International Journal of Bifurcation and Chaos, Vol. 11, No. 5 (2001) 1359-1380

(c) World Scientific Publishing Company

\title{
TIME-FREQUENCY ANALYSIS OF CLASSICAL TRAJECTORIES OF POLYATOMIC MOLECULES
}

\author{
LUZ V. VELA-AREVALO* and STEPHEN WIGGINS ${ }^{\dagger}$ \\ ${ }^{*}$ Control and Dynamical Systems, \\ ${ }^{\dagger}$ Applied Mechanics and Control and Dynamical Systems, \\ 107-81 Caltech, Pasadena, CA 91125, USA
}

Received June 20, 2000; Revised July 25, 2000

\begin{abstract}
We present a new method of frequency analysis for Hamiltonian Systems of 3 degrees of freedom and more. The method is based on the concept of instantaneous frequency extracted numerically from the continuous wavelet transform of the trajectories. Knowing the time-evolution of the frequencies of a given trajectory, we can define a frequency map, resonances, and diffusion in frequency space as an indication of chaos. The time-frequency analysis method is applied to the Baggott Hamiltonian to characterize the global dynamics and the structure of the phase space in terms of resonance channels. This 3-degree-of-freedom system results from the classical version of the quantum Hamiltonian for the water molecule given by Baggott [1988]. Since another first integral of the motion exists, the so-called Polyad number, the system can be reduced to 2 degrees of freedom. The dynamics is therefore simplified and we give a complete characterization of the phase space, and at the same time we could validate the results of the time-frequency analysis.
\end{abstract}

\section{Introduction}

The method of frequency analysis has been used as a numerical tool to study the global dynamics of systems of 3 degrees of freedom and more. By the computation of the fundamental frequencies associated to trajectories, this method allows us to determine the existence of quasiperiodic and chaotic zones in the phase space of a given dynamical system. The method was introduced by Laskar to study the chaotic dynamics of the Solar System [1990], and since then it has been widely used in celestial mechanics and galactic dynamics. Also, frequency analysis has been applied to Hamiltonian systems arising from the classical study of polyatomic molecules [Losada et al., 1998; Martens et al., 1987; vonMilczewski et al., 1997].

The frequencies associated to trajectories play an important role in the description of the dynamics of systems that are completely or nearly integrable. In this work we introduce a new method of timefrequency analysis based on wavelets that will allow us to define the instantaneous frequencies of trajectories for a large class of dynamical systems that were not tractable with standard frequency analysis. The innovation of the time-frequency analysis that we present is the assignment of frequencies that evolve in time. With this approach we are able to determine intrinsic resonances without any reference to the unperturbed Hamiltonian function or the frequency of the periodic perturbation. This new method will allow us to analyze trajectories of strongly nonintegrable systems as well.

Consider first an integrable Hamiltonian system with $n$ degrees of freedom (dof), then in theory it is possible to define action-angle coordinates $(I, \theta)$ in such a way that the Hamiltonian function can be 
written as

$$
H(I, \theta)=H_{0}(I)
$$

and the equations of motion can be easily solved,

$$
\begin{array}{ll}
\dot{I}_{k}=0 & \Rightarrow I_{k}=\text { constant }, \\
\dot{\theta}_{k}=\frac{\partial H}{\partial I_{k}}(I)=\omega_{k}(I) & \Rightarrow \theta_{k}(t)=\omega_{k}(I) t+\theta_{k}^{0}, \quad k=1, \ldots, n .
\end{array}
$$

The function $I \rightarrow \omega(I)$ is called the frequency map. If the frequency map is invertible, we can use the values of the frequencies $\omega_{k}$ as the coordinates instead of the actions $I_{k}$.

Therefore, the dynamics of completely integrable systems consists of quasiperiodic trajectories: the actions are constants of the motion and the angles are linear functions of time. All trajectories lie on invariant tori that are characterized by the fundamental frequencies $\omega_{k}$.

If the frequency vector $\omega=\left(\omega_{1}, \ldots, \omega_{n}\right)$ satisfies a Diophantine condition then the KAM theorem assures that the corresponding torus is not destroyed under small perturbations, and the trajectories are still quasiperiodic. Thus, the frequency vector can be used to characterize most of the trajectories of near-integrable systems, the quasiperiodic trajectories that remain after the perturbation.

Laskar's approach of frequency analysis consists of the numerical computation of the frequencies associated to a trajectory, therefore rendering a definition of the frequency map inherent of the solutions instead of the Hamiltonian function. With frequency analysis one can identify and completely characterize quasiperiodic trajectories (including of course periodic trajectories.) The method of Laskar for frequency analysis is based on Fourier analysis, more precisely on the windowed Fourier transform. Since the Fourier transform alone cannot account for any transition in time of the spectral information, the treatment of chaotic trajectories is not clearly substantiated. The idea of Laskar was to assume that the chaotic trajectories behave close to quasiperiodic in short intervals of time and to compute the frequency in successive intervals; the nature of chaotic trajectories arises from the variation of the computed frequencies. Laskar's method can be viewed in terms of the Gabor transform, where a continuous time parameter is introduced besides the frequency parameter. The time param- eter of the Gabor transform controls a sliding time window that localizes the trajectory around this parameter; however, Laskar uses disjoint intervals of time, i.e. the time parameter is sampled to obtain successive intervals.

Wavelet analysis provides better localization in time of the spectral information of a trajectory than the Gabor transform due to automatic adjustment of the size of the time window according to the frequency. This capability of the wavelet transform permits the definition and computation of timevarying frequencies. Therefore this method is much more suitable for chaotic trajectories.

The method of time-frequency analysis based on wavelets is such that:

1. it does not require near integrability, and

2 . it does not require that the system be in actionangle variables.

All rigorous notions of resonance in the mathematical literature are based on frequencies in some unperturbed or uncoupled systems. Our method enables us to define frequencies, and therefore resonances in strongly coupled and nonintegrable systems.

With the wavelet approach of time-frequency analysis, we can introduce a new definition of diffusion of a chaotic trajectory that accounts for the variation of the fundamental frequencies at all times, in contrast to Laskar's definition that uses the difference of frequencies in two successive intervals of time.

As an application of the method, we performed time-frequency analysis of the Baggott Hamiltonian.

The Baggott Hamiltonian is a quantum model of the water molecule which was originally developed by Baggott [1988] using spectroscopic methods. It is a three-mode vibrational Hamiltonian featuring 1:1 Darling-Dennison resonance between the stretch modes and $2: 1$ Fermi resonance between 
the bend and stretches, where the symmetric $x, K$ constraints have been relaxed to obtain better fitting to the experimental data. It has been used extensively in the literature in classical, semiclassical and quantum contexts. The same kind of model has been used for the description of triatomic symmetric molecules such as $\mathrm{D}_{2} \mathrm{O}, \mathrm{NO}_{2}, \mathrm{ClO}_{2}, \mathrm{O}_{3}$ and $\mathrm{H}_{2} \mathrm{~S}$ [Lu \& Kellman, 1997]. In these models there exists a constant of the motion known as the Polyad number, which will translate into an independent first integral in the classical regime.

The classical version of the Baggott Hamiltonian is obtained using the Heisenberg correspondence principle. It is a 3-dof Hamiltonian given in action-angle variables, and as the quantum version, contains $1: 1$ and $2: 1$ resonance couplings. The existence of the Polyad number allows the reduction of the system to 2-dof, and the global dynamics can be studied more easily.

In this work, we were interested in the case of Polyad number $P=34.5$, which corresponds to the quantum Polyad 16. This value is used in [Keshavamurthy \& Ezra, 1997] to study the quantum-classical correspondence of highly exited states of the molecule.

In [Keshavamurthy \& Ezra, 1997], a description of the classical phase space was given in terms of resonant two-dimensional tori and resonance channels calculated with the Chirikov resonance analysis. This procedure uses the integrable limits of the Hamiltonian obtained when only one resonance coupling is considered. The location of the resonance channels was the basis for the classification of the eigenstates of the Baggott Hamiltonian.

With the method of time-frequency analysis presented here, we are able to locate exactly the resonance channels and to show their angular dependence. Therefore, the Chirikov analysis gives only an approximation and the treatment of the Baggott Hamiltonian as a near integrable one is not so obvious.

We can also identify chaotic trajectories that are trapped temporarily around a resonant channel, suggesting the concept of time-varying resonances. This notion can explain how the energy of the system is distributed and how it is transported along the resonances during the evolution in time.

With the information of the resonances channels and the diffusion of the trajectories, we give a complete characterization of the dynamics in the phase space.
The organization of this paper is as follows: In the second section, we give a general description of the frequency analysis method based on Fourier analysis, as proposed by Laskar. In Sec. 3 we introduce the definition of time-varying frequency, and describe how wavelet analysis can be used for the computation of the evolution in time of the frequency. In Sec. 4 we present the Baggott Hamiltonian, and describe the reduction of the system to 2 degrees of freedom. The frequency analysis based on wavelets was used to analyze the classical trajectories of this system, this is presented in Sec. 5 together with the description of how the resonance structures determine the dynamics of the system. We also identify the chaotic zones in the phase space by numerically computing the diffusion of the system. The conclusion is found in Sec. 6. In the Appendix, we describe the transformations to obtain the 2-dof Baggott Hamiltonian, and outline the procedure for the computation of the frequency map.

\section{Laskar's Frequency Analysis}

The frequency analysis method implemented by Laskar [Laskar et al., 1992, 1993] is based on the fact that the fundamental frequencies of quasiperiodic motion remain constant in time. It uses Fourier analysis to obtain good approximations of quasiperiodic solutions by finite series of complex exponentials of the form $\sum_{j=0}^{N} c_{j} e^{2 \pi i \omega_{j} t}, c_{j} \in \mathbb{C}$.

For a nearly integrable system of $n$ degrees of freedom in the action-angle-like coordinates $(I, \theta)$, $I=\left(I_{1}, \ldots, I_{n}\right)$ and $\theta=\left(\theta_{1}, \ldots, \theta_{n}\right)$, Laskar's frequency analysis consists of the numerical computation of the fundamental frequency $\omega_{k}$ associated to each degree of freedom $z_{k}=\sqrt{2 I_{k}} e^{i \theta_{k}}$ of a given trajectory $\left(z_{1}, z_{2}, \ldots, z_{n}\right)(t)$, and of the approximation of the solution by an iterative scheme, giving

$$
z_{k}(t) \approx c_{1} e^{2 \pi i \omega_{k} t}+\sum_{j=2}^{N} c_{k}^{j} e^{2 \pi i \omega_{k}^{j} t} .
$$

If the given solution is quasiperiodic, a good approximation can be achieved and the value of the frequency vector $\omega=\left(\omega_{1}, \ldots, \omega_{n}\right)$ is close to the actual rotation vector of the trajectory. Thus, with this method we can determine and analyze quasiperiodic motions in the phase space. For instance, one can identify resonant regions when we have a set of initial conditions for which their 
frequency vector $\omega$ satisfies a resonance equation $\mathbf{k} \cdot \omega=0$ for some integer vector $\mathbf{k}$.

On the other hand, if the trajectory is chaotic, the approximation with a quasiperiodic series is compromised due to the evolution in time of the fundamental frequencies. The main idea of Laskar's approach when dealing with chaotic trajectories is to compute the fundamental frequencies in time intervals of the form $[\tau, \tau+T]$ for different values of $\tau$, and observe how they evolve. This approach has been used extensively in the literature: Chaotic regions are thus detected when the frequencies associated to a set of initial conditions vary greatly with respect to time and with respect to initial conditions. However, the selection of $\tau$ and $T$ is a very sensitive part of the procedure, because we have to assume that the trajectory is regular enough in each interval $[\tau, \tau+T]$ to obtain reliable information from the complex exponential expansions.

In this work, we will make use of the concept of instantaneous frequency based on wavelet analysis to obtain the evolution with respect to time of the frequencies associated to trajectories. This time-frequency analysis based on wavelets will be described in Sec. 3. Here we outline the method of Laskar as a motivation, and refer the reader to [Laskar et al., 1992, 1993] for a detailed description and the numerical implementation; also see [Laskar, 1999] for the analysis of the algorithm and its convergence.

To describe the frequency analysis method based on Fourier analysis, let us see how it works for a periodic function $f(t)=a e^{i 2 \pi \omega_{0} t}, a \in \mathbb{C}$, $t \in[-T, T]$.

The Fourier series of $f$ is given by

$$
\tilde{f}(t)=\sum_{-\infty}^{\infty} c_{n} e^{i \pi n t / T}
$$

where the coefficients are

$$
c_{n}=\left\langle f, e^{i \pi n t / T}\right\rangle
$$

(the inner product between two $2 T$-periodic functions is defined by $\left.\langle f, g\rangle=(1 / 2 T) \int_{-T}^{T} f(t) \bar{g}(t) d t\right)$.

For this particular $f$, the expression for the Fourier coefficients is given by

$$
c_{n}=a \frac{\sin \pi\left(2 \omega_{0}-n / T\right) T}{\pi\left(2 \omega_{0}-n / T\right) T} ;
$$

then the frequency $\omega_{0}$ is approximated by $n / 2 T$, where $n$ is the integer for which $c_{n}$ has maximum amplitude. Note that the error of this approximation is $1 / 2 T$.

With this method, it has to be assumed that the original function $f$ is $2 T$-periodic for the validity of the Fourier expansion.

The main idea behind Laskar's method allows us to disregard this assumption, considering that the exact value of the frequency $\omega_{0}$ can be recovered as the maximum of the function

$$
\phi(s)=\left|\left\langle f, e^{i 2 \pi s t}\right\rangle\right|=|a|\left|\frac{\sin 2 \pi\left(\omega_{0} q-s\right) T}{2 \pi\left(\omega_{0}-s\right) T}\right| .
$$

For the quasiperiodic function $f(t)=a_{1} e^{i \omega_{1} t}+$ $a_{2} e^{i \omega_{2} t}$, we proceed in a similar way. The maximum of

$\phi(s)=\left|\left\langle f, e^{i 2 \pi s t}\right\rangle\right|=\left|a_{1}\left\langle e^{i \omega_{1} t}, e^{i s t}\right\rangle+a_{2}\left\langle e^{i \omega_{2} t}, e^{i s t}\right\rangle\right|$

will be close to the frequency with greater amplitude, slightly distorted by the existence of the other frequency. The precision is good when the two frequencies are sufficiently far apart.

Thus, the procedure to find the fundamental frequency of a quasiperiodic function $f$ is to find the maximum of the function

$$
\phi(s)=\left|\left\langle f, e^{i 2 \pi s t}\right\rangle\right| .
$$

Note that with this method, instead of using the functions $e^{i n \pi / T}$ for the approximation of $f$, the basis functions have been changed to a more suitable set of exponentials $e^{i 2 \pi s t}$.

\section{Time-Frequency Analysis Using Wavelets}

Fourier Analysis yields a frequency representation of a time-dependent function or signal. However, Fourier methods require that the original function is periodic or quasiperiodic. Furthermore, frequency representation does not reflect at all any change in time of the spectral information of the function. Several attempts have been made to obtain a timefrequency representation of functions, most of them having their origin in signal processing. Researchers in that field have addressed the concept of instantaneous frequency from the representation of a function $f$ in the form

$$
f(t)=a(t) e^{i \phi(t)},
$$

and then defining the frequency as the derivative of the phase function $\phi$ (up to a factor of $2 \pi$ ). 
However, this representation is far from unique. See [Carmona et al., 1998] for a more detailed discussion.

Wavelet analysis has been a successful alternative to Fourier analysis for the representation of functions that possess time-varying frequencies. The innovation of this approach is the introduction of a second parameter that localizes in time the spectral properties of the analyzed function in a convenient way.

In this method, the idea is to express a function $f(t)$ in terms of basis functions constructed as translations and dilations of a so-called mother wavelet $\psi$ :

$$
\psi_{a, b}(t)=a^{-1 / 2} \psi\left(\frac{t-b}{a}\right) .
$$

The function $\psi \in L^{2}(\mathbb{R})$ must satisfy the admissibility condition $0<c_{\psi}=2 \pi \int_{-\infty}^{\infty}\left(|\hat{\psi}(\omega)|^{2} /|\omega|\right) d \omega<$ $\infty$, where $\hat{\psi}$ is the Fourier transform of $\psi$; to be useful the mother wavelet has to have compact support or to decay to 0 rapidly for $t \rightarrow \infty$ and $t \rightarrow-\infty$.

The coefficients of this expansion are given by the wavelet transform of $f$, defined as

$L_{\psi} f(a, b)=\left\langle f, \psi_{a, b}\right\rangle=a^{-1 / 2} \int_{-\infty}^{\infty} f(t) \bar{\psi}\left(\frac{t-b}{a}\right) d t$,

for $a>0$ and $b \in \mathbb{R}$.

The wavelet transform can be viewed as a timefrequency representation of $f$ in the following sense: If the wavelet $\psi$ has compact support, the parameter $b$ shifts the wavelets so that the local information of $f$ around the time $t=b$ is contained in $L_{\psi} f(a, b)$; the parameter $a$, called scale, is directly related to the frequency, therefore $L_{\psi} f(a, b)$ gives the frequency content of $f$ over a small interval of time around $t=b$. The wavelet transform can also be viewed as a function of the time variable $b$ and frequency variable (actually scale variable) $a$.

We want to consider local information of $f$ in time intervals that yield spectral information on any desirable range of frequencies (or frequency band.) For high-frequency spectral information, the time interval should be small; for low-frequency spectral information, the time interval should be wide. The wavelet transform possesses this property, called the "zoom-in" and "zoom-out" capabilities. This fact will be clear from the determination of the time window and frequency window on which the wavelet transform is localized; for this we need to introduce some concepts.
The center and radius of the wavelet are defined (respectively) by

$$
t^{*}=\frac{1}{\|\psi\|_{2}^{2}} \int_{-\infty}^{\infty} x|\psi(x)|^{2} d x
$$

and $\quad \Delta_{\psi}=\frac{1}{\|\psi\|_{2}}\left(\int_{-\infty}^{\infty}\left(x-t^{*}\right)^{2}|\psi(x)|^{2} d x\right)^{1 / 2}$.

Assume $\psi$ and its Fourier transform $\hat{\psi}$ are wavelets, with centers and radius $t^{*}, \omega^{*}, \Delta_{\psi}, \Delta_{\hat{\psi}}$. Then, the wavelet transform $L_{\psi} f(a, b)$ localizes the function within a time window of the form

$$
A=\left[b+a t^{*}-a \Delta_{\psi}, \quad b+a t^{*}+a \Delta_{\psi}\right] .
$$

Since the value of the frequency is proportional to $1 / a$, the time window automatically narrows for high frequency ( $a$ small) and widens for low frequency ( $a$ large); this is the "zoom in" and "zoom out" capabilities that we mentioned above.

Now, let $\eta(\omega)=\hat{\psi}\left(\omega+\omega^{*}\right)$, the center of $\eta$ is 0 and its radius is $\Delta_{\hat{\psi}}$. Using Parseval's identity, we obtain that

$L_{\psi} f(a, b)=\frac{a|a|^{-1 / 2}}{2 \pi} \int_{-\infty}^{\infty} \hat{f}(t) e^{i \omega b} \bar{\eta}\left(a\left(\omega-\frac{\omega^{*}}{a}\right)\right) d \omega$

then the same quantity $L_{\psi} f(a, b)$ gives localized information of the spectrum $\hat{f}(\omega)$ within a frequency window

$$
B=\left[\frac{\omega^{*}}{a}-\frac{\Delta_{\hat{\psi}}}{a}, \quad \frac{\omega^{*}}{a}+\frac{\Delta_{\hat{\psi}}}{a}\right],
$$

(with the exception of multiplication by $a|a|^{1 / 2} / 2 \pi$ and a linear phase shift of $\left.e^{i \omega b}\right)$.

Therefore, $L_{\psi} f(a, b)$ localizes $f$ in the timefrequency window $A \times B$.

The wavelet transform produces a complex surface as a function of the variables $a$ and $b$. A common representation of this surface is a density plot of the modulus of $L_{\psi} f(a, b)$, with $b$ as the horizontal axis (time) and $\ln (a)$ as the vertical axis. However, since the frequency is proportional to the inverse of the scale $(1 / a)$ we opted for the frequency $\omega$ as the vertical axis. 

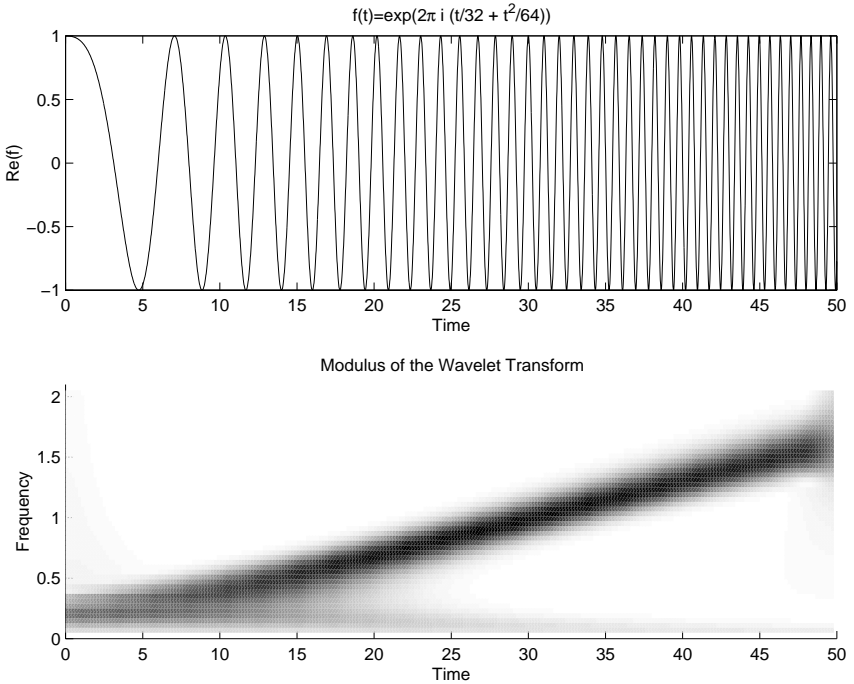

Fig. 1. Density plot of the modulus of the continuous wavelet transform of a function with time-varying frequency. The darker gray zones correspond to larger modulus of the wavelet transform.

Figure 1 shows the real part of a function with linearly increasing frequency, and the density plot of its wavelet transform. Note the exact correspondence of the local frequency content of $f$ with the modulus of its wavelet transform. This fact is the basis for the time-frequency analysis that we propose in this work.

The mother wavelet that we use throughout this work is the Morlet-Grossman wavelet [Carmona et al., 1998], given by

$$
\psi(t)=\frac{1}{\sigma \sqrt{2 \pi}} e^{2 \pi i \lambda t} e^{-t^{2} / 2 \sigma^{2}} .
$$

The parameters $\lambda$ and $\sigma$ can be tuned to improve the resolution, in our case we used mostly the values 1 and 2 respectively.

Let us describe now how we can use the wavelet transform to recover the variation in time of the frequency of a function $f$.

As a first example, consider a periodic function $f(t)=e^{i 2 \pi \omega_{0} t}$, for which the wavelet transform can be obtained analytically as

$$
\begin{aligned}
L_{\psi} f(a, b) & =a^{-1 / 2} \int_{-\infty}^{\infty} e^{i 2 \pi \omega_{0} t} \bar{\psi}\left(\frac{t-b}{a}\right) d t \\
& =a^{1 / 2} \int_{-\infty}^{\infty} e^{i 2 \pi \omega_{0} a s} e^{i 2 \pi \omega_{0} b} \bar{\psi}(s) d s \\
& =a^{1 / 2} e^{i 2 \pi \omega_{0} b} \overline{\hat{\psi}}\left(\omega_{0} a\right),
\end{aligned}
$$

where $\hat{\psi}$ is the Fourier transform of $\psi$. As we should expect the modulus of $L_{\psi} f(a, b)$ depends only on the scale $a$, and it is independent of the time variable $b$.

Particularly for the Morlet-Grossman wavelet, the modulus of this transform is given by

$$
\eta(a)=\left|L_{\psi} f(a, b)\right|=\sqrt{a} \overline{\hat{\psi}}\left(\omega_{0} a\right),
$$

with $\hat{\psi}(\omega)=e^{-2 \pi^{2} \sigma^{2}(\omega-\lambda)^{2}} . \quad \eta(a)$ has a global maximum, in other words, $\hat{\psi}$ is well localized in frequency. The maximum is at $a \omega_{0}=(1 / 2)(\lambda+$ $\left.\sqrt{\lambda^{2}+\left(1 / 2 \pi^{2} \sigma^{2}\right)}\right)$. Therefore, we can define the frequency variable (for the Morlet-Grossman wavelet) as

$$
\omega=\frac{1}{2 a}\left(\lambda+\sqrt{\lambda^{2}+\frac{1}{2 \pi^{2} \sigma^{2}}}\right),
$$

and the value of the frequency $\omega_{0}$ can be recovered from the scale $a$ that maximizes the modulus of the wavelet transform.

The power of the wavelet transform is that this procedure can be extended immediately for oscillatory functions for which the value of the frequency is not constant in time. The maximum of the modulus of the wavelet transform at each $b$ gives the frequency content of $f$ over a time window about $b$ by means of the frequency variable given before. So we can determine the frequency of $f$ in terms of the time variable $t=b$.

However, one has to remember that the localization in both time and frequency is limited by the uncertainty principle (see for instance [Gasquet \& Witomski, 1998, p. 197].)

\section{Baggott Hamiltonian}

The classical version of the quantum Hamiltonian for the water molecule by Baggott [1988] is given by

$$
\begin{aligned}
H= & H_{0}+H^{1: 1}+H^{2: 2}+H_{1}^{2: 1}+H_{2}^{2: 1}, \\
H_{0}= & \Omega_{s}\left(I_{1}+I_{2}\right)+\Omega_{b} I_{3}+\alpha_{s}\left(I_{1}^{2}+I_{2}^{2}\right) \\
& +\alpha_{b} I_{3}^{2}+\varepsilon_{s s} I_{1} I_{2}+\varepsilon_{s b} I_{3}\left(I_{1}+I_{2}\right), \\
H^{1: 1}= & \left(\beta_{12}+\lambda^{\prime}\left(I_{1}+I_{2}\right)\right. \\
& \left.+\lambda^{\prime \prime} I_{3}\right)\left(I_{1} I_{2}\right)^{1 / 2} \cos \left(\theta_{1}-\theta_{2}\right), \\
H^{2: 2}= & \beta_{22} I_{1} I_{2} \cos 2\left(\theta_{1}-\theta_{2}\right) \\
H_{1}^{2: 1}= & \beta_{s b}\left(I_{1} I_{3}^{2}\right)^{1 / 2} \cos \left(\theta_{1}-2 \theta_{3}\right), \\
H_{2}^{2: 1}= & \beta_{s b}\left(I_{2} I_{3}^{2}\right)^{1 / 2} \cos \left(\theta_{2}-2 \theta_{3}\right),
\end{aligned}
$$


Table 1. Parameters of the Baggott Hamiltonian.

\begin{tabular}{cc}
\hline$\Omega_{s}$ & $3885.57\left(\mathrm{~cm}^{-1}\right)$ \\
$\Omega_{b}$ & 1651.72 \\
$\alpha_{s}$ & -81.99 \\
$\alpha_{b}$ & -18.91 \\
$\varepsilon_{s s}$ & -12.17 \\
$\varepsilon_{s b}$ & -19.12 \\
$\beta_{12}$ & -112.96 \\
$\lambda^{\prime}$ & 6.04 \\
$\lambda^{\prime \prime}$ & -0.16 \\
$\beta_{22}$ & -1.82 \\
$\beta_{s b}$ & 18.79 \\
\hline
\end{tabular}

where $\left(I_{1}, I_{2}, I_{3}, \theta_{1}, \theta_{2}, \theta_{3}\right)$ are action-angle coordinates.

The Baggott Hamiltonian is a 3-degree-offreedom (dof) system exhibiting 2:1 resonance between the bend and stretch modes, and $1: 1$ and $2: 2$ resonances between the two stretch modes. There is a permutation symmetry between the indices 1 and 2, and also note that the Hamiltonian is not differentiable at $I_{1}=0$ or $I_{2}=0$. The values of the parameters are obtained to fit the experimental spectra. We used here the notation in [Keshavamurthy \& Ezra, 1997]. See Table 1.

The system possesses a first integral which is the classical expression of the quantum Polyad number of the system. It is given by

$$
P=2\left(I_{1}+I_{2}\right)+I_{3} .
$$

This constant of motion $P$ is related to the quantum Polyad P by $P=2 \mathrm{P}+5 / 2$ [Keshavamurthy \& Ezra, 1997]. Note Eq. (2) implies that the values of the actions are bounded: $0<I_{1}<P / 2,0<I_{2}<P / 2$ and $0 \leq I_{3}<P$.

In this work, we study the Polyad number $P=34.5$, that corresponds to the quantum Polyad number $\mathrm{P}=16$. This is the same value used in [Keshavamurthy \& Ezra, 1997] to study highly excited states of the molecule. We will use timefrequency analysis based on wavelets to give a more accurate and complete characterization of the phase space for this Polyad number.

Taking the angles $\theta_{1}=\theta_{2}=\theta_{3}=0$, the intersection of the energy surfaces $H=H_{0}$ with the plane $P=34.5$ can be drawn as contours projected

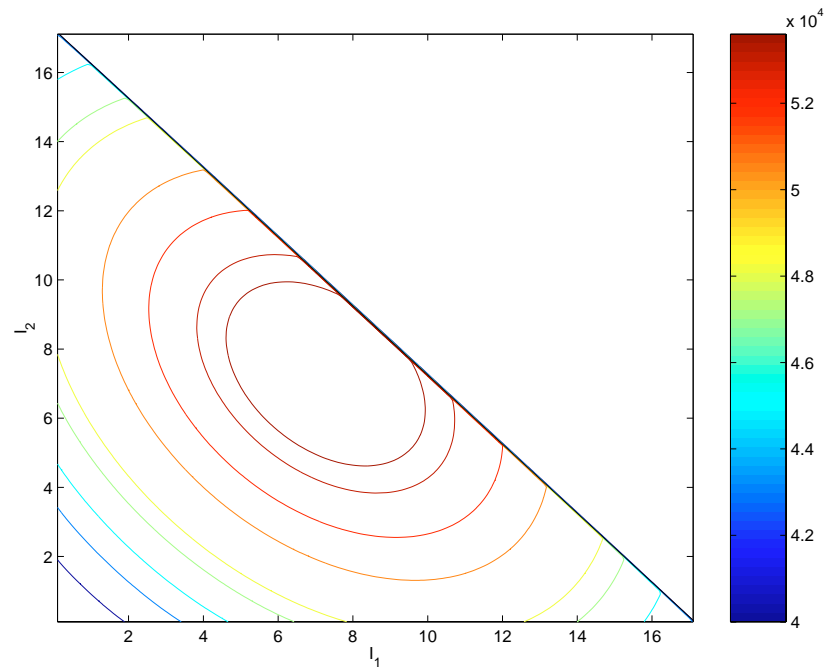

Fig. 2. Energy levels for a slice of the phase space corresponding to $\theta_{1}=\theta_{2}=\theta_{3}=0$, projected onto the action plane $\left(I_{1}, I_{2}\right)$, with $I_{3}=P-2\left(I_{1}+I_{2}\right)$, and $P=34.5$.

to the plane $I_{1}, I_{2}$, since $I_{3}$ is obtained from Eq. (2). These contours are represented in Fig. 2.

Equation (2) will allow us to reduce the Baggott Hamiltonian to a 2-dof system. Via successive canonical transformations we obtain first the Hamiltonian in normal coordinates, and then a system in action-angle variables - $\left(N_{1}, N_{2}\right.$, $\left.N_{3}, \psi_{1}, \psi_{2}, \psi_{3}\right)$ for which the third action satisfies

$$
N_{3}=P \text {, }
$$

and the conjugate angle $\psi_{3}$ does not appear explicitly in the Hamiltonian, as we should expect. The details of these transformations can be found in the Appendix.

Therefore, for a fixed value of $P$, the system can be considered as a 2 -dof system in the variables $\left(N_{1}, N_{2}, \psi_{1}, \psi_{2}\right)$, and afterwards we can solve for the third angle $\psi_{3}$.

Table 2. Parameters of the 2-dof Hamiltonian (in $\mathrm{cm}^{-1}$ ) for $P=34.5$.

\begin{tabular}{lc}
\hline$\alpha_{1}$ & $-112.96-0.16 \mathrm{P}=-118.48$ \\
$\alpha_{2}$ & $525.65+56.44 \mathrm{P}=2472.83$ \\
$\alpha_{3}$ & $1651.72 \mathrm{P}-18.91 \mathrm{P}^{2}=34476.71$ \\
$\beta_{1}$ & 26.5731 \\
$\beta_{2}$ & -76.8150 \\
$\beta_{3}$ & 73.1750 \\
$\beta_{4}$ & 79.5350 \\
$\beta_{5}$ & -78.7125 \\
\hline
\end{tabular}



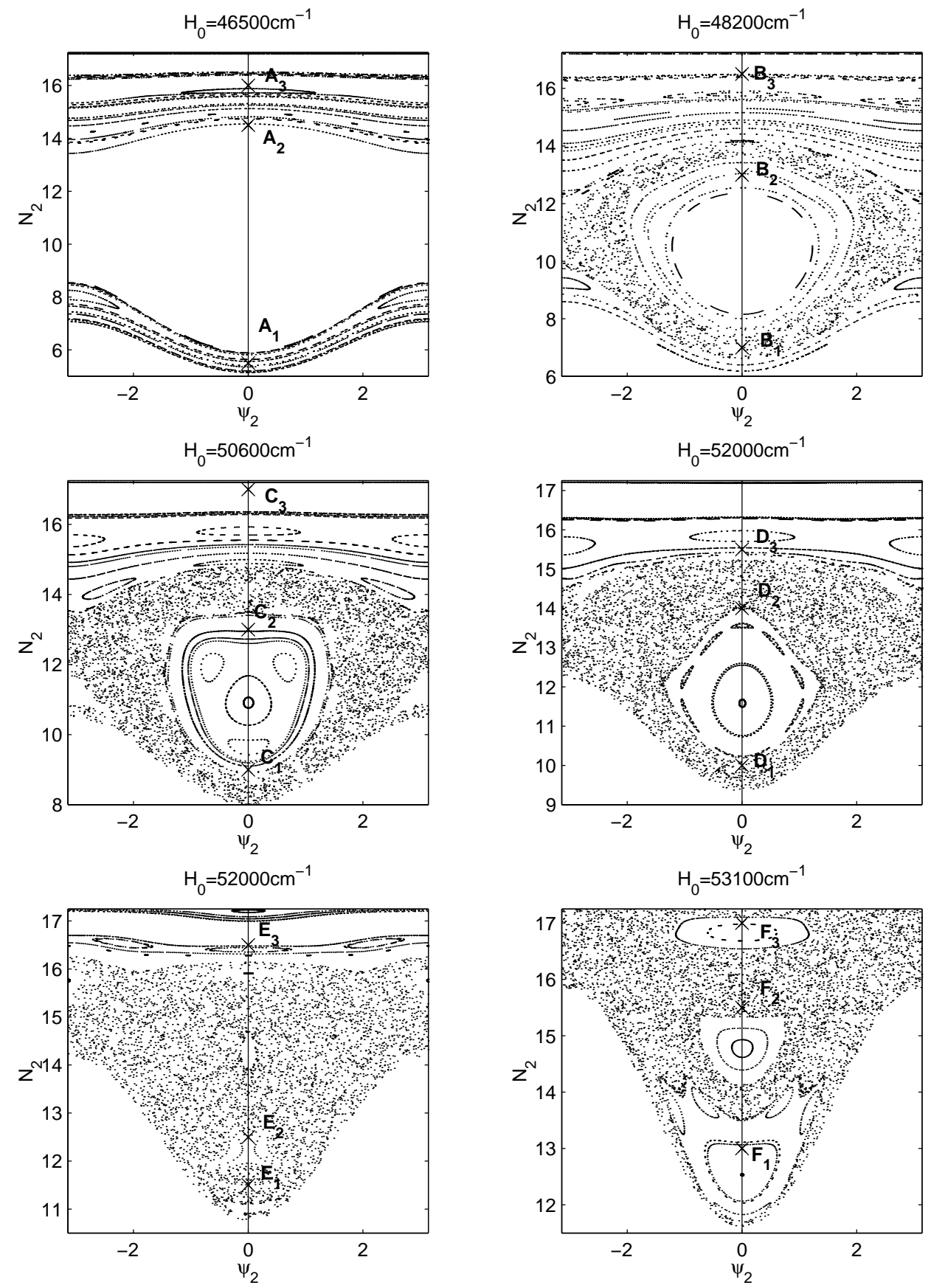

Fig. 3. Poincaré sections for $P=34.5, \psi_{1}=0, \dot{\psi}_{1}>0$.

The 2-dof Hamiltonian is given by

$$
\begin{aligned}
H= & \alpha_{1} N_{1}+\alpha_{2} N_{2}+\alpha_{3}+\beta_{3} N_{1}^{2}+\beta_{4} N_{1} N_{2}+\beta_{5} N_{2}^{2} \\
& +\beta_{1} \sqrt{N_{1}+N_{2}}\left(-2 N_{2}+P\right) \cos \psi_{2} \\
& -\beta_{2}\left(N_{1}^{2}+N_{1} N_{2}\right) \cos 2 \psi_{1} .
\end{aligned}
$$

For the Polyad number $P=34.5$ the values of the parameters are given in Table 2 .
Since the Baggott Hamiltonian can be reduced to a 2-dof system, it is possible to construct Poincaré maps to obtain a global view of the dynamics for $P$ fixed and a particular value of the energy $H=H_{0}$.

In action-angle coordinates $\left(N_{1}, N_{2}, \psi_{1}, \psi_{2}\right)$, the Poincaré section we defined is:

$$
\begin{aligned}
\Sigma & =\left\{\left(N_{2}, \psi_{2}\right): H\left(N_{1}, N_{2}, \psi_{1}, \psi_{2}\right)\right. \\
& \left.=H_{0}, \psi_{1}=0, \dot{\psi}_{1}>0\right\} .
\end{aligned}
$$


The plots of the Poincaré maps for $P=34.5$ and different values of the energy $H_{0}$ are shown in Fig. 3. We can observe alternation between chaotic and regular motions, and the chaotic motion predominates for higher values of the energy.

\section{Wavelet Time-Frequency Analysis of the Baggott Hamiltonian}

For completely integrable Hamiltonians in actionangle variables $H(I, \theta)=H_{0}(I)$ the frequency map is defined by

$$
I \rightarrow \omega(I)=\frac{\partial H_{0}}{\partial I} .
$$

This map characterizes trajectories for nearly integrable systems as well, since the nonresonant tori are preserved under small perturbations, according to the KAM theorem.

The computation of time-varying frequencies enables the assignment of frequencies even for nonnearly-integrable cases. The time-frequency analysis we present relies on a frequency map computed numerically with the wavelet approach described in Sec. 3.

We perform time-frequency analysis of the Baggott Hamiltonian for the case $P=34.5$.

To define the frequency map we proceed as follows: Constrain the initial conditions to a particular slice of the phase space given by

$$
\begin{gathered}
0<I_{1}<P / 2, \quad 0<I_{2}<P / 2, \quad I_{1}+I_{2} \leq P / 2, \\
I_{3}=P-2\left(I_{1}+I_{2}\right), \\
\theta_{1}=\theta_{2}=\theta_{3}=0,
\end{gathered}
$$

(see Fig. 2).

For a given initial condition $\left(I_{1}^{0}, I_{2}^{0}\right)$ in this slice, we obtain numerically the trajectories over the time interval $[0, T]$ and express them in complex coordinates $z_{k}(t)=\sqrt{2 I_{k}(t)} e^{i \theta_{k}(t)}, k=1,2,3$, for $t \in[0, T]$. We then obtain the evolution in time of the associated fundamental frequencies $\omega_{k}(t)$ for $t \in[0, T]$, by computing the maximum of the modulus of the wavelet transform (see Sec. 3). The frequency map is then defined as:

$$
\left(I_{1}^{0}, I_{2}^{0}, I_{3}^{0}\right) \longrightarrow\left(\omega_{1}, \omega_{2}, \omega_{3}\right)(t), t \in[0, T] .
$$

A more detailed description of the computation of the frequency map can be found in the Appendix.

Some examples of time-frequency analysis for the Baggott Hamiltonian are shown in Fig. 4.
The reduction to 2-dof of the Baggott Hamiltonian allows us to compare the frequency map analysis with the Poincaré map for each trajectory. In this way we can decide whether a given trajectory is quasiperiodic or chaotic, and see that there is an exact correspondence with time-frequency analysis: quasiperiodic trajectories have frequencies that remain constant in time, whereas chaotic trajectories have frequencies with large variation in time.

Time-frequency analysis based on wavelets has the obvious advantage over Poincaré maps of being applicable to any number of degrees of freedom; besides it provides a quantitative as well as a qualitative method to characterize quasiperiodic, resonant and chaotic motions. The advantage over the Laskar approach of frequency analysis, described in Sec. 2, is the improved resolution of the evolution in time of the frequencies associated with the trajectory. Due to the "zoom in" and "zoom out" capabilities of wavelets (see Sec. 3) we do not have to assume slow transition of the frequencies or to propose a priori the size of the interval in which the trajectory behaves close to quasiperiodic, which is a sensitive part of the Laskar approach.

With time-frequency analysis, quasiperiodic trajectories can be identified due to the small deviation of $\omega_{k}(t)$ from its mean value $\tilde{\omega}_{k}$. This mean value is actually the value that we would obtain with the method of Laskar; thus we can recover the results obtained with that approach. We can define a "mean frequency vector" $\left(\tilde{\omega}_{1}, \tilde{\omega}_{2}, \tilde{\omega}_{3}\right)$ that corresponds to the fundamental frequency in the case of quasiperiodic solutions.

With this in mind we can compute the Arnold web, which is the mapping of the actions to the frequency ratios:

$$
\left(I_{1}^{0}, I_{2}^{0}\right) \longrightarrow\left(\frac{\tilde{\omega}_{1}}{\tilde{\omega}_{3}}, \frac{\tilde{\omega}_{2}}{\tilde{\omega}_{3}}\right)
$$

For a grid of evenly spaced initial conditions $\left(I_{1}^{0}, I_{2}^{0}\right)$ in the slice (4), we performed timefrequency analysis and plotted the Arnold web in Fig. 5.

We can observe the lines corresponding to resonance equations:

$$
k_{1} \tilde{\omega}_{1}+k_{2} \tilde{\omega}_{2}+k_{3} \tilde{\omega}_{3}=0,
$$

for some integers $k_{1}, k_{2}, k_{3}$. Therefore, the main resonances found are the ones we expected for the form of the Hamiltonian: 1:1 resonance between 
a)

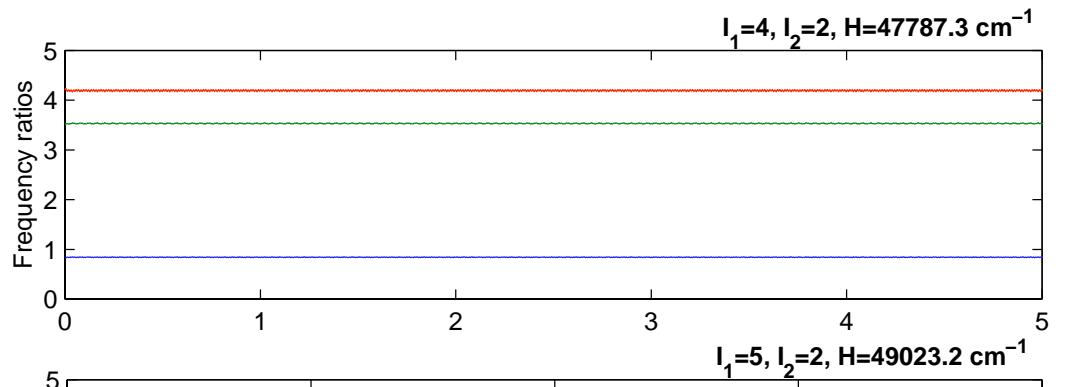

b)

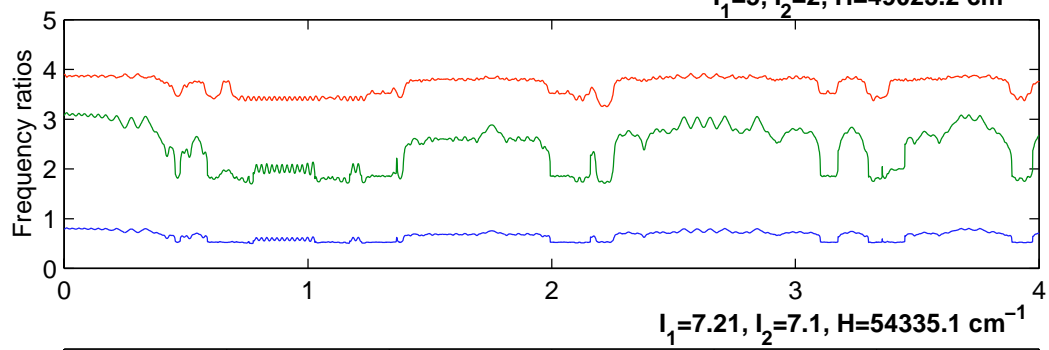

c)

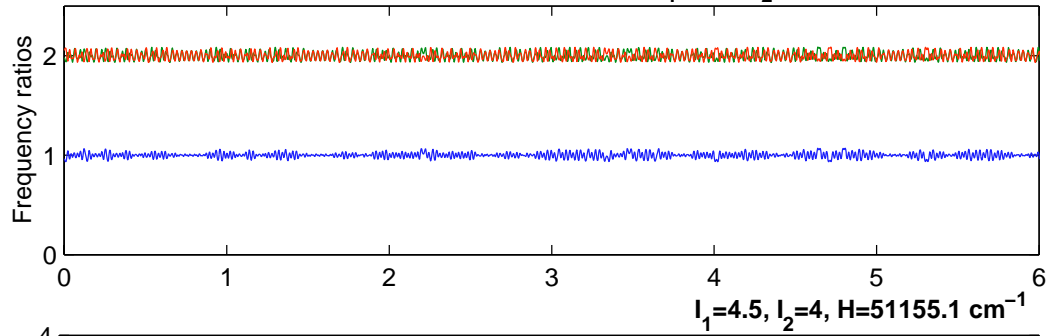

d)

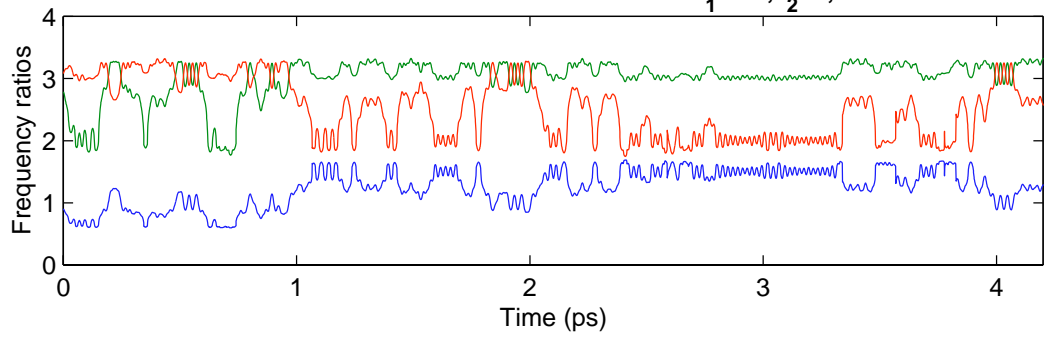

Fig. 4. Time-frequency analysis for some trajectories of the Baggott Hamiltonian, $\omega_{1} / \omega_{2}$ is blue, $\omega_{1} / \omega_{3}$ is green, and $\omega_{2} / \omega_{3}$ is red, (a) corresponds to a quasiperiodic trajectory, meanwhile (b)-(d) correspond to chaotic trajectories.

$I_{1}$ and $I_{2}$ (stretches), and 2:1 resonance between $I_{1}$ and $I_{3}$, and between $I_{2}$ and $I_{3}$ (stretch-bend). We found also higher order resonances, all of them intersecting at the same point. See Fig. 5.

Note in Fig. 5 that the resonance line $\tilde{\omega}_{1}=\tilde{\omega}_{2}$ (the $45^{\circ}$ line) is rather isolated, yielding to the conjecture that this resonance exists but it does not have a large effect on the quasiperiodic motion nearby. On the contrary, the $2: 1$ resonance lines (the vertical line $\tilde{\omega}_{1} / \tilde{\omega}_{3}=2$ and the horizontal line $\tilde{\omega}_{2} / \tilde{\omega}_{3}=2$ ) are surrounded by many dots, indicating that the $2: 1$ resonance has a strong effect on the motion for this particular slice of the phase space.

With time-frequency analysis we can locate the resonant trajectories, i.e. initial conditions for which their mean frequencies satisfy a given resonance equation up to certain accuracy. Inverting the frequency map, we can find the resonant regions in the phase space; the results for the Baggott Hamiltonian are presented in Fig. 6. We can see that the predominant resonances are in three areas, one corresponding to $1: 1$ resonance on the symmetry line $I_{1}=I_{2}$, and two regions corresponding to the $2: 1$ resonances. There are also higher order resonances, as indicated in the Fig. 5.

We also plotted in Fig. 6 some invariant twodimensional stable tori with initial conditions in the slice. The tori were found as stable fixed points of the Poincaré maps of the 2-dof system (3): these points correspond to stable periodic orbits for the four-dimensional system, and these periodic orbits correspond to stable 2-tori of the six-dimensional Baggott Hamiltonian (1). We can see in Fig. 6 that the resonant tori are at the center of the so-called resonance channels, i.e. initial conditions strongly affected by the existence of the resonance. 


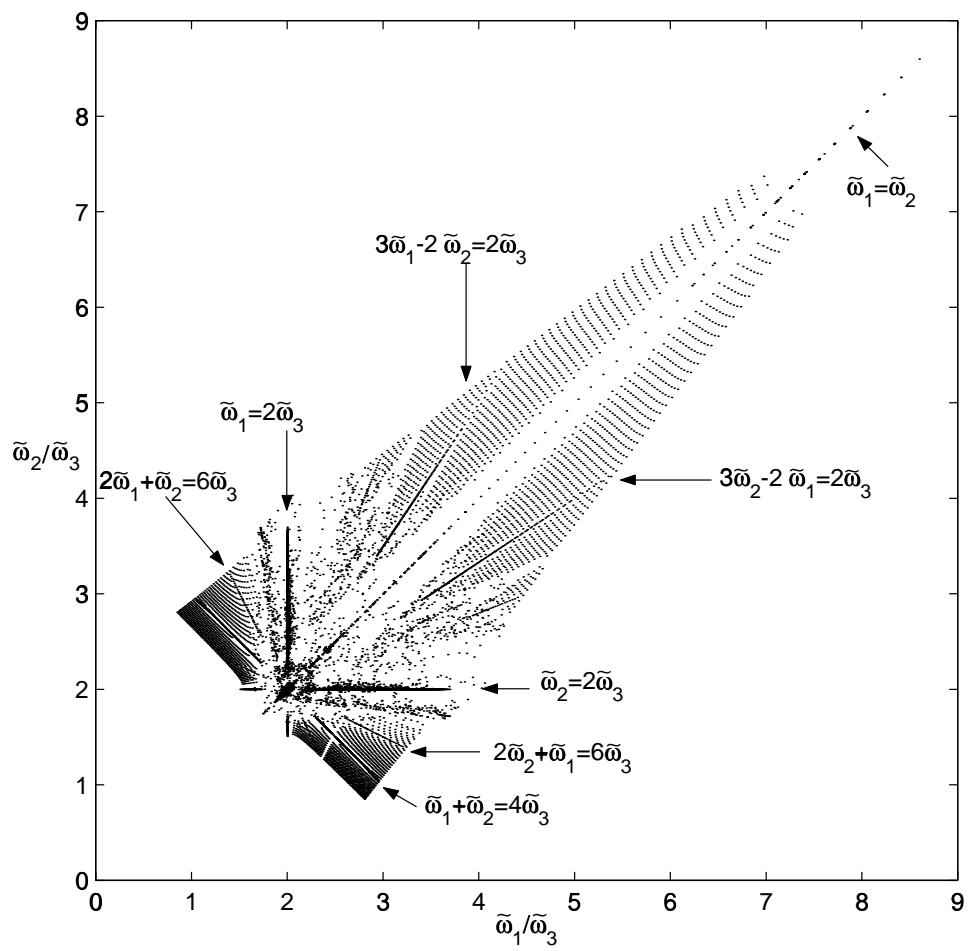

Fig. 5. Arnold web of the Baggott Hamiltonian for $P=34.5$.

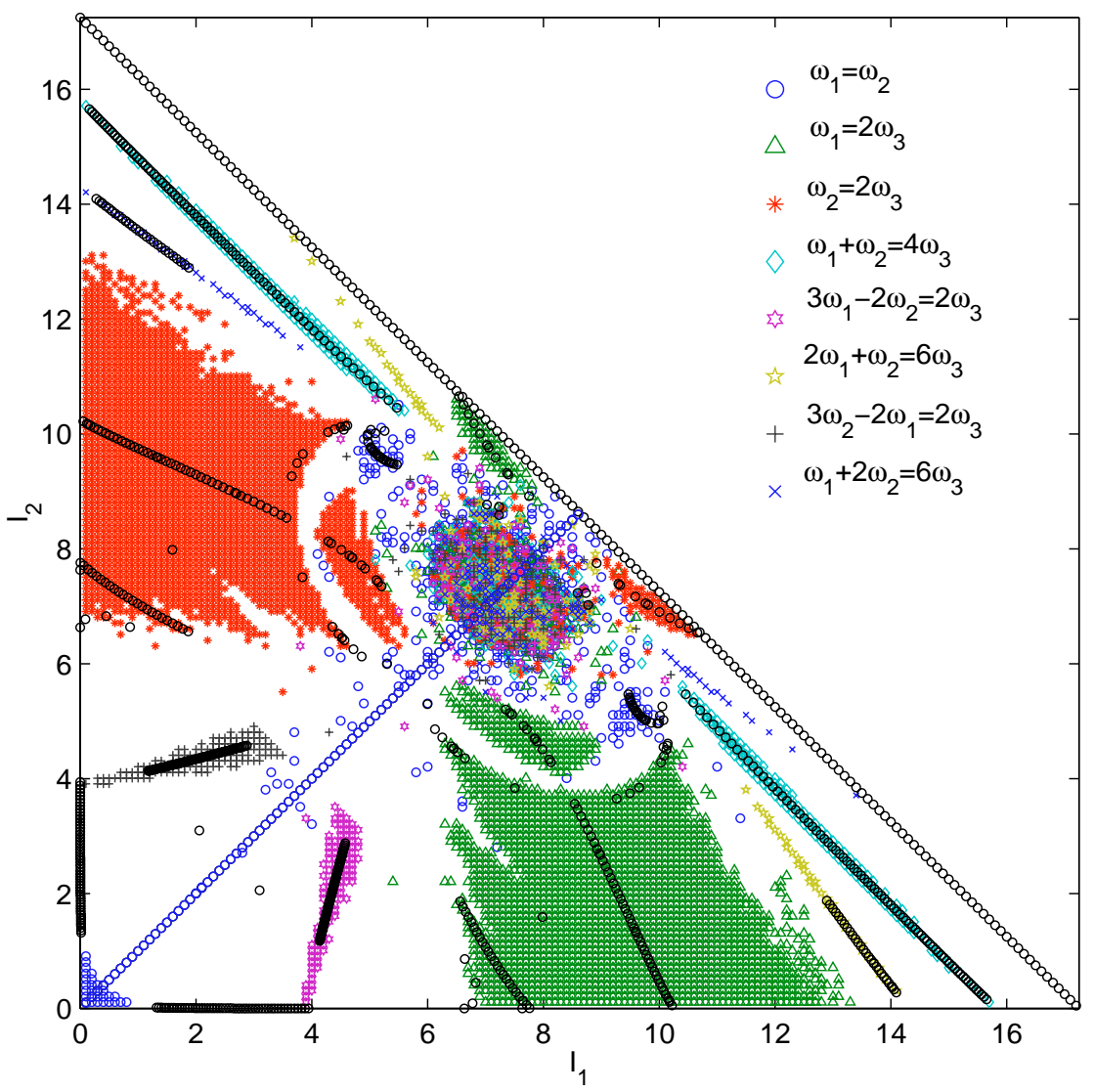

Fig. 6. Resonance channels. Stable 2-tori are indicated in black circles. 
In [Keshavamurthy \& Ezra, 1997], they consider the $H_{0}$ part of (1) as the unperturbed Hamiltonian, and the remaining terms as small perturbations. Then, they define the frequencies as in the near-integrable case, i.e. as the partial derivatives of $H_{0}$ with respect to the actions. These frequencies were used to determine the classical resonances. They also obtained the resonance channels using the Chirikov analysis, a procedure that uses the integrable limits of the Hamiltonian, i.e. when some terms are neglected.

Figure 1 in [Keshavamurthy \& Ezra, 1997] has some similarities with our Fig. 6, regarding the location of the main resonances, the resonant tori, and the resonance channels. However, the results of the time-frequency analysis we present correspond to the full Hamiltonian (1), and not only to the integrable approximations used in [Keshavamurthy \& Ezra, 1997].

We can observe in Fig. 5 that all the resonances intersect at the same point. This can be seen also in Fig. 6, since around the point $\left(I_{1}, I_{2}\right)=(7.2703,7.2703)$ the resonance channels overlap. We will show in Sec. 5.2 that there is a periodic orbit passing through this point, that corresponds to the intersection of the two main resonances, $1: 1$ and $2: 1$.

\section{Diffusion}

The concept of diffusion of a trajectory is used generally to describe a drift in the action variables and characterize unstable motion in the phase space. This concept can be translated to a variation in frequencies using the method of time-frequency analysis.

We explained before that the chaotic motion is characterized by the variation in time of the frequencies with respect to their mean value. The size of this variation is closely related to the drift of the trajectory in action space, this is something that we can check again by looking at the Poincaré map. If the trajectory is strongly chaotic, i.e. if it fills up a big area of the Poincare section in a short time, there is a large deviation of the frequencies from their mean value. This motivated our definition of diffusion of a trajectory in terms of the frequencies:

Definition 1. For a trajectory with initial conditions $\left(I^{0}, \theta^{0}\right)$, time-frequency analysis provides the evolution in time of its fundamental frequencies: $\omega_{1}, \omega_{2}, \omega_{3}$ in $[0, T]$. Then we define the diffusion of the trajectory over the interval $[0, T]$ by

$$
\operatorname{diffusion}\left(I^{0}, \theta^{0}\right)=\operatorname{dev}_{1}+\operatorname{dev}_{2}+\operatorname{dev}_{3},
$$

where

$$
\operatorname{dev}_{k}=\frac{1}{T} \int_{0}^{T}\left|\omega_{k}(t)-\tilde{\omega}_{k}\right| d t
$$

Laskar [1993] used an approximation of the second derivative of the frequency map with respect to initial conditions as an indicator of the chaotic behavior of the trajectory. He also showed some examples for which this approximation is proportional to the derivative of the frequency map with respect to time, calculated as the difference of the frequency in two successive intervals of time. Arguing that this relation is characteristic of diffusion, he used these approximations interchangeably as a measure of the diffusion of the trajectory in frequency space.

Our definition of diffusion also makes use of this correspondence between chaotic trajectories and variation of the frequencies. However, we include all the spectral information of the trajectory in the interval $[0, T]$, given by the frequency map. Meanwhile, Laskar used only the "mean frequencies" in two successive intervals. It is clear that the better resolution we achieve with frequency analysis based on wavelets yields a more dynamic indicator of the diffusion.

We compute the diffusion of the Baggott Hamiltonian in the slice (4) with the frequency map described above. Therefore, to each initial condition in the slice, we associate the diffusion and plot a density graph corresponding to this slice of the phase space. The results of these computations are shown in Fig. 7. This figure renders the symmetry of the system with respect to $I_{1}$ and $I_{2}$, (recall that the initial conditions were taken symmetric, $\theta_{1}=\theta_{2}$.) The quasiperiodic areas (low diffusion) and chaotic areas (high diffusion) are well distinguished. We can observe that for low values of the energy (see also Fig. 2) most initial conditions have low diffusion; however, as the energy increases, we observe alternation between low and high diffusion, showing the coexistence of regular motion and strongly chaotic motion. When the energy is close to the maximum (around the point $\left.\left(I_{1}, I_{2}\right)=(7.2703,7.2703)\right)$ large diffusion predominates but it is not as large as in some other regions, indicating that all trajectories are chaotic but they do not have much room to wander. 
Figure 7 confirms the results in Fig. 6: The resonance channels have zero or very low diffusion, that is, the values of the frequencies remain fairly constant in time and therefore the motion is quasiperiodic in those areas. The symmetry line $I_{1}=I_{2}$, corresponding to the $1: 1$ resonance, is surrounded by quasiperiodic motion for low values of the energy (up to $46500 \mathrm{~cm}^{-1}$ ). For higher values of the energy the symmetry line is surrounded by trajectories with large diffusion (strongly chaotic) therefore, the $1: 1$ resonance channel is very thin in this slice, basically it includes only the line $I_{1}=I_{2}$. On the contrary, the $2: 1$ resonance channels correspond to a large region with low diffusion, then the resonant 2-tori are surrounded by quasiperiodic trajectories strongly affected by the resonance.

All the results in the diffusion plot correspond exactly to the analysis of the Poincaré maps (Fig. 3.) We have picked some points in Fig. 3 on the line $\psi_{2}=0$, and the same points have been plotted in the original coordinates in Fig. 7 with their corresponding energy contours. This allows us to compare the dynamics in both figures, as we do in the following. Note we only plotted points with $I_{1}<I_{2}$; the symmetric points with $I_{1}>I_{2}$ correspond to a different Poincaré section and the analysis is analogous.

For low values of the energy $H_{0}$, all the trajectories are quasiperiodic (see points $\mathrm{A}_{1}, \mathrm{~A}_{2}, \mathrm{~A}_{3}$.) When $H_{0}$ increases, chaotic motion appears (see the point $\mathrm{B}_{1}$ ) alternating with regular motion.

In the Poincaré sections we can observe quasiperiodic trajectories for large values of $N_{2}$ (points $\mathrm{B}_{3}, \mathrm{C}_{3}, \mathrm{D}_{3}$,) some of them in resonant islands (points $\mathrm{A}_{3}, \mathrm{E}_{3}$.) For all these trajectories, the value of $I_{3}$ is small, or equivalently $I_{1}+I_{2} \approx P / 2$; physically this corresponds to initial conditions with the bend mode close to equilibrium.

The 2:1 resonance channel corresponds to the large regular area at the center of the Poincaré sections, appearing for the first time around $48800 \mathrm{~cm}^{-1}$. This regular area remains up to values of the energy around $53100 \mathrm{~cm}^{-1}$, for instance between the points $\mathrm{C}_{1}$ and $\mathrm{C}_{2}$ and between the points $\mathrm{D}_{1}$ and $\mathrm{D}_{2}$; the closed curves we see in the Poincaré sections between these points correspond

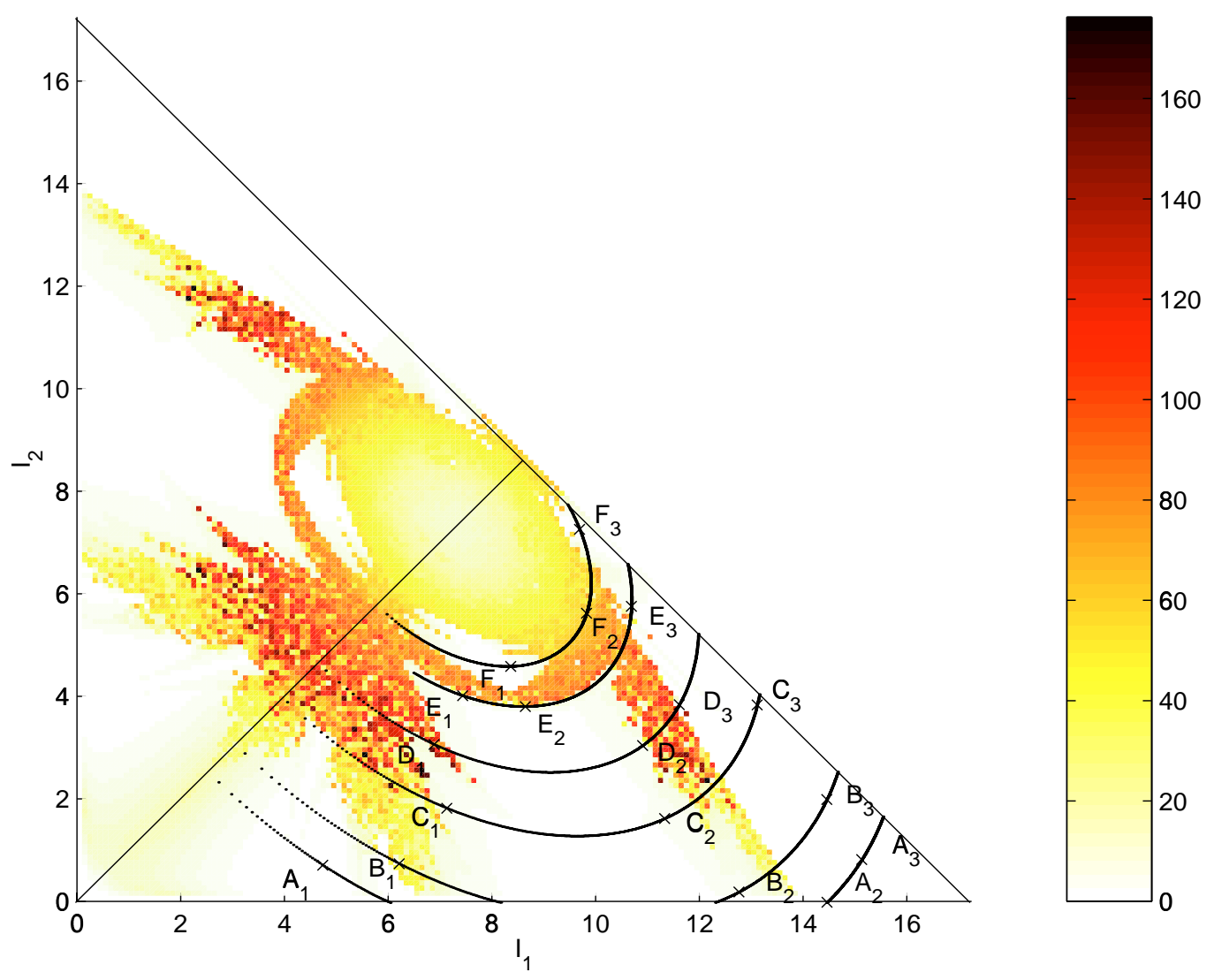

Fig. 7. Diffusion plot of the Baggott Hamiltonian. The points marked on the energy contours correspond to the points in the Poincaré sections of Fig. 3. 
to quasiperiodic trajectories lying on invariant 3 -tori of the 3-dof system, these trajectories form the resonance channel, since they have frequencies close to the $2: 1$ resonance.

Note in both Figs. 6 and 7 that the boundary of the 2:1 resonance channel has some "fingers" or gaps. These are consequence of the alternation between small regular islands and chaotic regions that we can observe in the Poincaré sections. The islands correspond to higher order resonances that we did not plot.

At the point $\mathrm{E}_{2}$ in Fig. 7 the $2: 1$ resonance channel breaks. This can be seen also in the Poincaré section for $H_{0}=53100 \mathrm{~cm}^{-1}$ : Instead of the regular region, there is a saddle point surrounded by chaotic trajectories. The corresponding 2 -torus is unstable.

For $H_{0}=53600 \mathrm{~cm}^{-1}$ we find several resonances, the point $\mathrm{F}_{1}$ is in the $2: 1$ resonance between $I_{1}$ and $I_{3}$, and the point $\mathrm{F}_{3}$ is in the $2: 1$ resonance between $I_{2}$ and $I_{3}$. There is also a small quasiperiodic region with $1: 1$ resonance. All these resonances alternate with chaotic trajectories.

The quasiperiodic regions disappear completely around $53900 \mathrm{~cm}^{-1}$, and from here to the maximum $\left(54340 \mathrm{~cm}^{-1}\right)$ all trajectories have large diffusion, corresponding to Poincaré sections filled with chaotic trajectories.

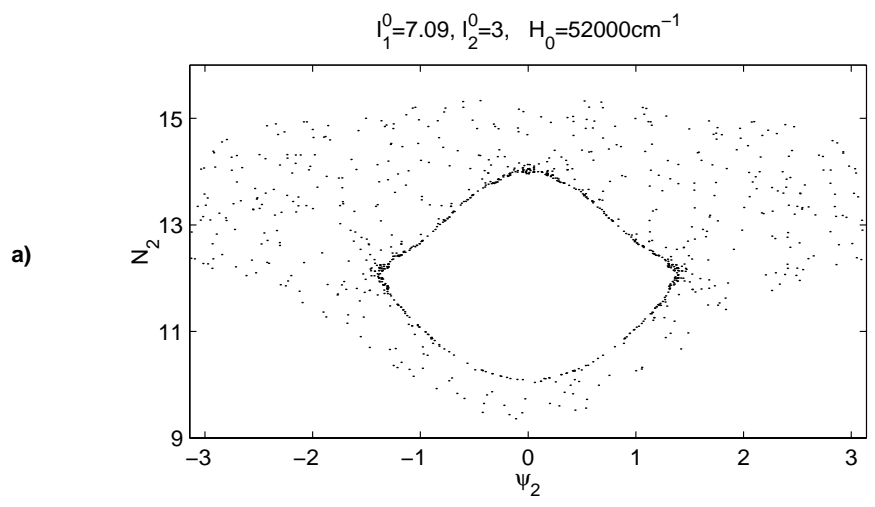

b)

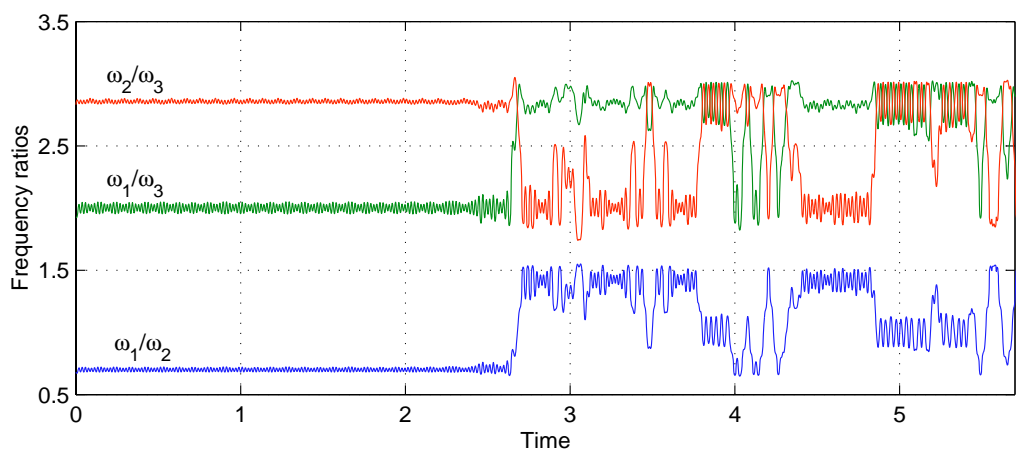

c)

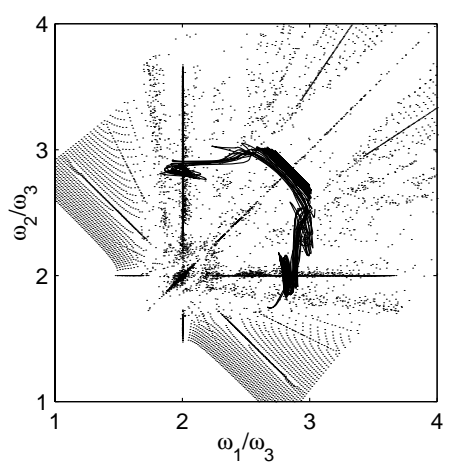

Fig. 8. One chaotic trajectory showing first low diffusion around the $2: 1$ resonance, and then wandering around the main resonance channels. See text for explanation. 
Comparing the resonance plot and the diffusion plot (Figs. 6 and 7) we observe some quasiperiodic areas which are not close to resonance (for instance the region around points $\mathrm{B}_{3}$ and $\mathrm{C}_{3}$ ). These areas may act as boundaries for the diffusion of trajectories. To explain this with an example, in Fig. 8 we plot different representations of one chaotic trajectory: (a) the Poincaré map, (b) its frequency evolution in time, and (c) the frequency ratios (dark line) on top of the Arnold web (dots). Note there is an accumulation of points around a resonant island of the Poincaré section, corresponding in (b) to the beginning where the trajectory is close to the $2: 1$ resonance. After certain time the trajectory leaves this resonance, but we can observe that it wanders around the resonances $2: 1$ and $1: 1$. This is more clear in (c), the trajectory passes by the three main resonance lines; however, it remains around the point $\left(\tilde{\omega}_{1} / \tilde{\omega}_{3}, \tilde{\omega}_{2} / \tilde{\omega}_{3}\right)=(2,2)$, and never goes to the regions corresponding to quasiperiodic trajectories.

This particular trajectory is not the exception, it can be observed in many chaotic trajectories that their frequencies wander around the main resonances when they evolve in time. This suggests the concept of time-varying resonances, i.e. trajectories for which their frequencies are trapped temporarily around a resonance, but they leave for another resonance and so forth. Energy transport between the different modes of the motion can occur in this way via chaotic trajectories.

\subsection{Two more slices of the phase space}

All the analysis that we have done so far was for the particular slice of the phase space given in (4). We have shown how frequency analysis allows a complete description of the phase space for initial conditions in that particular slice.

To obtain a more complete analysis of the phase space, we consider two different slices, and perform time-frequency analysis on them with the same procedure as before. Figures 9 and 10 show the resonance channels and the diffusion plot for initial conditions evenly spaced in action space, and angles given by

$$
\theta_{1}^{0}=\pi, \quad \theta_{2}^{0}=0, \quad \theta_{3}^{0}=0
$$

and

$$
\theta_{1}^{0}=\pi, \quad \theta_{2}^{0}=\pi, \quad \theta_{3}^{0}=0,
$$

respectively.

Observe that Fig. 9 is no longer symmetric as a result of nonsymmetric initial conditions. In this slice we can observe that the $1: 1$ resonance between the stretches $\left(I_{1}\right.$ and $\left.I_{2}\right)$ is predominant, and the $2: 1$ resonance between $I_{1}$ and $I_{3}$ is not present. This shows how the Chirikov resonance analysis performed in [Keshavamurthy \& Ezra, 1997] disregards the angular dependence of the resonance channels. The structure of the phase space can hardly be understood with the integrable approximations of the Hamiltonian. The Baggott Hamiltonian cannot be treated as nearly integrable without losing important features of the dynamics.

In Fig. 10 the initial conditions are symmetric, however we can observe new higher order resonances occupying large regions of the slice, showing a different structure from that of (4).

\subsection{Some more phase space structure}

It is still interesting to look at the symmetry line $I_{1}=I_{2}$. This line corresponds to $N_{1}=0$ in the reduced coordinates (3) and is invariant under the 2-dof flow. In the full six-dimensional phase space, this invariant surface is two-dimensional when the energy is fixed.

We can study the flow on this surface by looking at the intersection of the set $N_{1}=0$ with a constant energy surface $H=H_{0}$. The intersection can be expressed as the invariant curve

$$
\begin{aligned}
Q_{1}= & \left\{\left(0, N_{2}, 0, \psi_{2}\right): \alpha_{3}+\alpha_{2} N_{2}+\beta_{5} N_{2}^{2}\right. \\
& \left.+\beta_{1} \sqrt{N_{2}}\left(-2 N_{2}+P\right) \cos \psi_{2}=H_{0}\right\},
\end{aligned}
$$

(where to avoid the indefinition of the angle $\psi_{1}$ for $N_{1}=0$, we have fixed it arbitrarily as 0 ). The curves for several values of $H_{0}$ can be observed in Fig. 11.

Note that the fixed points of the 2-dof system in $Q_{1}$ correspond to periodic orbits when the third angle $\psi_{3}$ is considered, therefore they are periodic orbits of the full 3-dof system (1). The fixed point at $\psi_{2}=0, N_{2}=14.5406$ is a center-saddle point for the 2-dof system, therefore the corresponding periodic orbit of the 3-dof system has a threedimensional center manifold, a two-dimensional unstable manifold and a two-dimensional stable manifold. Its coordinates in the original variables 

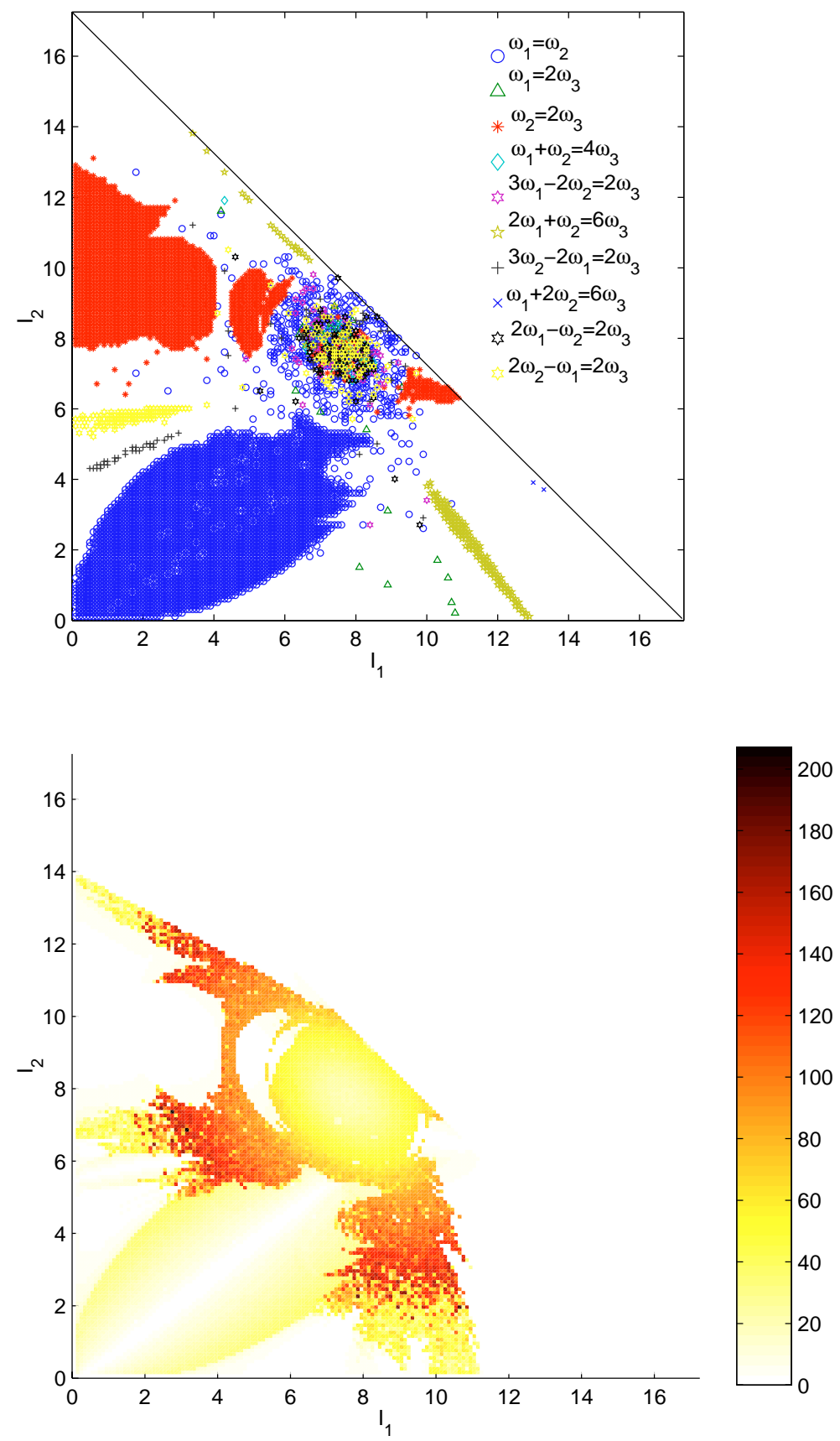

Fig. 9. Resonance channels and diffusion plot for the slice $\theta_{1}=\pi, \theta_{2}=\theta_{3}=0, I_{3}=P-2\left(I_{1}+I_{2}\right), P=34.5$.

are expressed as $I_{1}=I_{2}=N_{2} / 2=7.2703, I_{3}=$ $P-2 N_{2}, \theta_{1}=\theta_{2}=2 \psi_{3}, \theta_{3}=\psi_{3}$.

Furthermore, this periodic orbit can be found directly in the original coordinates (1) if we solve for the intersection of the resonance channels $1: 1$ and $2: 1$; that is, solving the equation $\dot{\theta}_{1}=2 \dot{\theta}_{3}$ for $I_{1}$ considering $I_{1}=I_{2}$ and $\theta_{1}=\theta_{2}=2 \theta_{3}$. We have to note that this periodic orbit is located at the global maximum of the energy $H$ restricted to the slice (4).
Since most of the curves $Q_{1}$ are closed $\left(\psi_{2}\right.$ is an angle in $[0,2 \pi])$ they correspond to two-dimensional tori in the six-dimensional system. Observing that the line $\psi_{2}=0$ in Fig. 11 corresponds exactly to the line $I_{1}=I_{2}$ with $I_{1}=N_{2} / 2$, then we can conclude that this line is foliated with invariant 2 -tori (with the exception of the periodic orbit we just mentioned above).

Another invariant surface of the 2-dof flow (3) is given by $N_{2}=P / 2$, since for this value $\dot{N}_{2}=0$. 

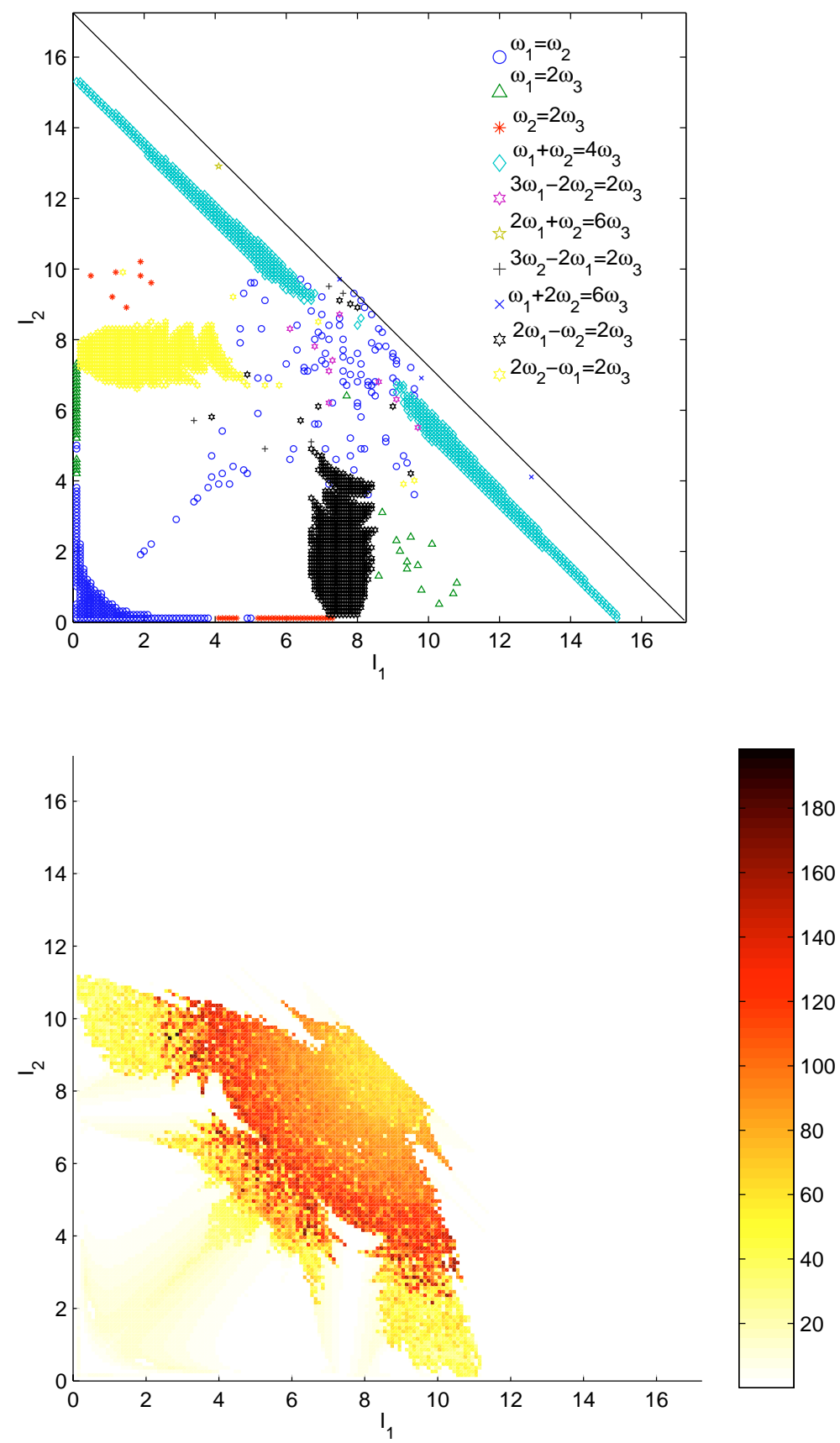

Fig. 10. Resonance channels and diffusion plot for the slice $\theta_{1}=\theta_{2}=\pi, \theta_{3}=0, I_{3}=P-2\left(I_{1}+I_{2}\right), P=34.5$.

This surface corresponds to the invariant set $I_{3}=0$ in the original coordinates (1), in other words, the bend mode is in equilibrium. This is the case of a system with only the 1:1 stretch coupling that can be reduced to 1-dof, therefore completely integrable. In [Xiao \& Kellman, 1989], this problem is studied and a complete characterization of the phase space in terms of $P$ is given.

The intersection of the surface $N_{2}=P / 2$ with a constant energy manifold can be written as

$$
\begin{aligned}
Q_{2}= & \left\{\left(N_{1}, \frac{P}{2}, \psi_{1}, \psi_{2}\right): \alpha_{3}+\alpha_{2} \frac{P}{2}\right. \\
& +\beta_{5} \frac{P^{2}}{4}+\left(\alpha_{1}+\frac{\beta_{4}}{2} P\right) N_{1}+\beta_{3} N_{1}^{2} \\
& \left.-\beta_{2} N_{1}\left(N_{1}+\frac{P}{2}\right) \cos 2 \psi_{1}=H_{0}\right\} .
\end{aligned}
$$

The intersection of this surface for a given value of $H_{0}$ with a plane $\psi_{2}=$ constant is represented by a curve in Fig. 12. 


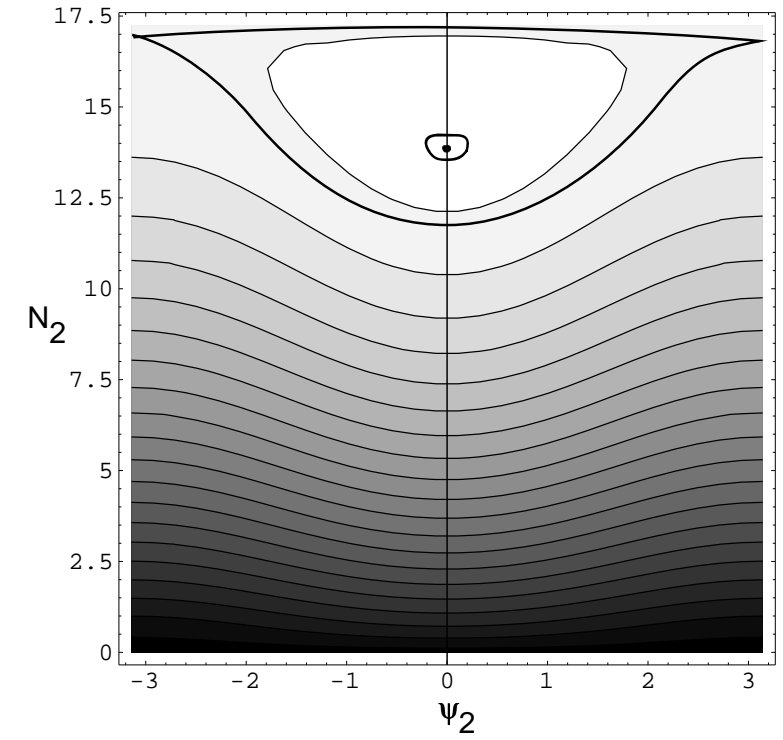

Fig. 11. Invariant curve $Q_{1}=\left\{N_{1}=0\right\}$. Each curve corresponds to a given value of the energy $H_{0}$.

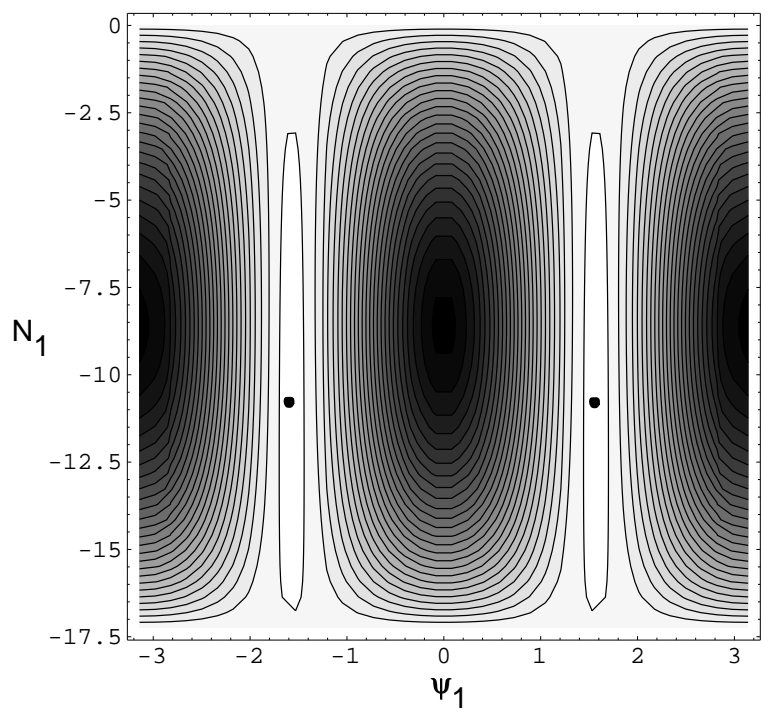

Fig. 12. Intersection of the invariant surfaces $Q_{2}=\left\{N_{2}=\right.$ $P / 2\}$ with a plane $\psi_{2}=$ constant, for different values of $H_{0}$.

The expression of $Q_{2}$ we obtained here corresponds exactly to the normal mode representation of the Hamiltonian in [Xiao \& Kellman, 1989]. In that work, they propose a reparametrization of the trajectories such that the phase space is a sphere with constant Polyad number. We refer the reader to that work for a detailed explanation of the bifurcations in the phase space.

We'll see that $Q_{2}$ is a two-dimensional manifold in the phase space. Since $I_{3}=0$, the conjugate angle $\theta_{3}$ must be fixed arbitrarily to avoid indefi- nition, say $\theta_{3}=0$. After the coordinate transformations, this implies $\psi_{3}=0$. For $H_{0}$ fixed, $Q_{2}$ is two-dimensional manifold when $\psi_{2}$ is considered. More precisely the closed curves in Fig. 12 are 2-tori for the 2-dof system. Since the third angle $\psi_{3}=0$, they are also 2-tori in the full six-dimensional phase space.

The fixed points in $Q_{2}$ are actually periodic orbits of the 2 -dof system $\left(\psi_{2} \in[0,2 \pi]\right)$. Again, since $\psi_{3}=0$, they are periodic orbits for the full six-dimensional system. The fixed points at $N_{1}=$ $-8.59, \psi_{1}=0, \pi$ are elliptic, then the corresponding periodic orbits are stable and they can be expressed in the original coordinates as $I_{1}=17.2499$, $I_{2}=0.00004, I_{3}=0, \theta_{1}=\theta_{2}=\psi_{2}, \theta_{3}=0$, and the symmetric point $I_{1}=0.00004, I_{2}=17.2499$.

In the intersection of $Q_{1}$ and $Q_{2}$ there is another periodic orbit, with coordinates $N_{1}=0, N_{2}=P / 2$, $\psi_{1}=0$ and $\psi_{2} \in[0,2 \pi]$. The coordinates of this orbit in the six-dimensional phase space are $I_{1}=I_{2}=P / 4, I_{3}=0, \theta_{1}=\theta_{2}=\psi_{2}, \theta_{3}=0$. This periodic orbit is unstable.

Some fixed points of (3) not lying in the invariant surfaces can be found by solving numerically the equations $\dot{\psi}_{1}=0, \dot{\psi}_{2}=0$ with $\psi_{1}=0, \pi$ and $\psi_{2}=0$. These points correspond to periodic orbits for the six-dimensional phase space, since the third angle $\psi_{3}$ is a parameter in $[0,2 \pi]$. Their coordinates in the original variables are $I_{1}=10.27, I_{2}=0.0009$, $I_{3}=13.9504, \theta_{1}=\theta_{2}=2 \psi_{3}, \theta_{3}=\psi_{3}$; and its corresponding symmetric point, $I_{1}=0.0009, I_{2}=10.27$; the orbits are stable.

\section{Conclusion}

The method of frequency analysis based on wavelets that we have presented is a good alternative to study a wide range of dynamical systems. Since wavelet analysis allows simultaneous localization in frequency and time, we can compute time-varying frequencies associated to trajectories, and in this way characterize the motion of systems with oscillatory trajectories.

Time-frequency analysis based on wavelets proved to be very suitable for the Baggott Hamiltonian due to the vibrational character of the solutions. For this system, the fundamental frequencies are obtained with great accuracy in the case of quasiperiodic solutions.

And due to the capability of adjusting the time window automatically for every frequency, with the 
wavelet approach we compute more reliable information about the variation in time of the frequencies of chaotic trajectories. This is the main advantage with respect to the frequency analysis of Laskar, where a fixed interval of time is used, disregarding the possibility of rapid transition in the frequencies.

Also, the definition of diffusion we present seems more adequate than the one that is given by Laskar. Since we are capable to compute the variation with respect to time of the frequencies, we obtain an indicator of the diffusion every time.

With frequency analysis based on wavelets we were able to characterize the phase space of the Baggott Hamiltonian for the Polyad number $P=$ 34.5. We have located the resonance channels, the quasiperiodic motion and the regions with large diffusion, producing an exact picture of the global dynamics of the system. We also showed the angular dependence of the resonance channels, concluding that the system cannot be treated as a nearly integrable one.

\section{Acknowledgments}

This research was partially supported by Consejo Nacional de Ciencia y Tecnologia (CONACYT México); and by the National Science Foundation Grant DMS-9704759.

\section{References}

Baggott, J. E. [1988] "Normal modes and local modes in $\mathrm{H}_{2} X$ : Beyond the $x, K$ relations," Molec. Phys. 65, 739-749.

Carmona, R., Hwang, W. \& Torrésani, B. [1998] Practical Time-Frequency Analysis: Gabor and Wavelet Transforms with an Implementation in $S$ (Academic Press).

Gasquet, C. \& Witomski, P. [1998] Fourier Analysis and Applications (Springer).

Keshavamurthy, S. \& Ezra, G. [1997] "Eigenstate assignments and the quantum-classical correspondence for highly excited vibrational states of the Baggott $\mathrm{H}_{2} \mathrm{O}$ Hamiltonian," J. Chem. Phys. 107, 156-179.

Laskar, J. [1990] "The chaotic motion of the solar system: A numerical estimate of the size of the chaotic zones," Icarus 88, 226-291.

Laskar, J., Froeschlé, C. \& Celletti, A. [1992] "The measure of chaos by the numerical analysis of the fundamental frequencies. Application to the standard mapping," Physica D56, 253-269.

Laskar, J. [1993] "Frequency analysis for multidimensional systems. Global dynamics and diffusion," Physica D67, 257-281.
Laskar, J. [1999] "Introduction to frequency map analysis," in Hamiltonian Systems with Three or More Degrees of Freedom, ed. Simo, C., pp. 134-150.

Losada, J. C., Estebaranz, J. M. \& Benito, R. M. [1998] "Local frequency analysis and the structure of classical phase space of the LiNC/LiCN molecular system," J. Chem. Phys. 108, 63-71.

Lu, Z. \& Kellman, M. E. [1997] "Phase space structure of triatomic molecules," J. Chem. Phys. 107, 1-15.

Martens, C., Davis, M. \& Ezra, G. [1987] "Local frequency analysis of chaotic motion in multidimensional systems: Energy transport and bottlenecks in planar OCS," Chem. Phys. Lett. 142, 519-528.

vonMilczewski, J., Farrelly, D. \& Uzer, T. [1997] "Frequency analysis of 3D electronic $1 / \mathrm{r}$ dynamics: Tuning between order and chaos," Phys. Rev. Lett. 78, 1436-1439.

Xiao, L. \& Kellman, M. E. [1989] "Unified semiclassical dynamics for molecular resonance spectra," J. Chem. Phys. 90, 6086-6098.

\section{Appendix A}

\section{Reduction to 2 degrees of freedom}

The following transformations of variables were performed first to obtain a normal form of the Hamiltonian (1), and then to reduce the system to 2 degrees of freedom using the Polyad number (2).

With complex coordinates defined by the canonical transformation

$$
z_{k}=\sqrt{2 I_{k}} e^{i \theta_{k}}, \quad \bar{z}_{k}=\sqrt{2 I_{k}} e^{-i \theta_{k}},
$$

the Hamiltonian function (1) can be rewritten as,

$$
H=H_{0}+H_{1}+H_{2},
$$

where

$$
\begin{aligned}
H_{0}= & \frac{\Omega_{s}}{2}\left(\left|z_{1}\right|^{2}+\left|z_{2}\right|^{2}\right)+\frac{\Omega_{b}}{2}\left|z_{3}\right|^{2}+\frac{\beta_{12}}{2} \operatorname{Re}\left(z_{1} \bar{z}_{2}\right) \\
H_{1}= & \frac{\beta_{s b}}{2^{3 / 2}}\left[\operatorname{Re}\left(z_{1} \bar{z}_{3}^{2}\right)+\operatorname{Re}\left(z_{2} \bar{z}_{3}^{2}\right)\right] \\
H_{2}= & \frac{\alpha_{s}}{4}\left(\left|z_{1}\right|^{4}+\left|z_{2}\right|^{4}\right)+\frac{\alpha_{b}}{4}\left|z_{3}\right|^{4}+\frac{\varepsilon_{s s}}{4}\left|z_{1}\right|^{2}\left|z_{2}\right|^{2} \\
& +\frac{\varepsilon_{s b}}{4}\left|z_{3}\right|^{2}\left(\left|z_{1}\right|^{2}+\left|z_{2}\right|^{2}\right) \\
& +\left(\frac{\lambda^{\prime}}{2}\left(\left|z_{1}\right|^{2}+\left|z_{2}\right|^{2}\right)+\frac{\lambda^{\prime \prime}}{2}\left|z_{3}\right|^{2}\right) \frac{1}{2} \operatorname{Re}\left(z_{1} \bar{z}_{2}\right) \\
& +\frac{\beta_{22}}{4} \operatorname{Re}\left[\left(z_{1} \bar{z}_{2}\right)^{2}\right] .
\end{aligned}
$$


And Eq. (2) in these coordinates is

$$
P=\left|z_{1}\right|^{2}+\left|z_{2}\right|^{2}+\frac{\left|z_{3}\right|^{2}}{2}
$$

In order to diagonalize the quadratic terms of this Hamiltonian, the following symplectic change of coordinates is defined,

$$
\begin{array}{lll}
z_{1}=\frac{1}{\sqrt{2}}\left(x_{1}+x_{2}\right), & \bar{z}_{1}=\frac{1}{\sqrt{2}}\left(\bar{x}_{1}+\bar{x}_{2}\right), \\
z_{2}=\frac{1}{\sqrt{2}}\left(x_{1}-x_{2}\right), & \bar{z}_{2}=\frac{1}{\sqrt{2}}\left(\bar{x}_{1}-\bar{x}_{2}\right), \\
z_{3}=x_{3}, & \bar{z}_{3}=\bar{x}_{3} .
\end{array}
$$

The expressions in the Hamiltonian become,

$$
\begin{aligned}
H_{0}= & \left(\frac{\Omega_{s}}{2}+\frac{\beta_{12}}{4}\right)\left|x_{1}\right|^{2}+\left(\frac{\Omega_{s}}{2}-\frac{\beta_{12}}{4}\right)\left|x_{2}\right|^{2} \\
& +\frac{\Omega_{b}}{2}\left|x_{3}\right|^{2}, \\
H_{1}= & \frac{\beta_{s b}}{2}\left[\operatorname{Re}\left(x_{1} \bar{x}_{3}^{2}\right)\right], \\
H_{2}= & \frac{\alpha_{s}}{8}\left[\left(\left|x_{1}\right|^{2}+\left|x_{2}\right|^{2}\right)^{2}+4\left(\operatorname{Re}\left(x_{1} \bar{x}_{2}\right)\right)^{2}\right] \\
& +\frac{\varepsilon_{s s}}{16}\left[\left(\left|x_{1}\right|^{2}+\left|x_{2}\right|^{2}\right)^{2}-4\left(\operatorname{Re}\left(x_{1} \bar{x}_{2}\right)\right)^{2}\right] \\
& +\frac{\varepsilon_{s b}}{4}\left|x_{3}\right|^{2}\left(\left|x_{1}\right|^{2}+\left|x_{2}\right|^{2}\right)+\frac{\alpha_{b}}{4}\left|x_{3}\right|^{4} \\
& +\left(\frac{\lambda^{\prime}}{2}\left(\left|x_{1}\right|^{2}+\left|x_{2}\right|^{2}\right)+\frac{\lambda^{\prime \prime}}{2}\left|x_{3}\right|^{2}\right) \\
& \times \frac{1}{4}\left(\left|x_{1}\right|^{2}-\left|x_{2}\right|^{2}\right) \\
& +\frac{\beta_{22}}{16}\left[\left(\left|x_{1}\right|^{2}-\left|x_{2}\right|^{2}\right)^{2}-4\left(\operatorname{Im}\left(x_{1} \bar{x}_{2}\right)\right)^{2}\right],
\end{aligned}
$$

and

$$
P=\left|x_{1}\right|^{2}+\left|x_{2}\right|^{2}+\frac{\left|x_{3}\right|^{2}}{2} .
$$

Action-angle variables can be defined by

$$
x_{k}=\sqrt{2 J_{k}} e^{i \phi_{k}}, \quad \bar{x}_{k}=\sqrt{2 J_{k}} e^{-i \phi_{k}},
$$

that yield to the following form of the Hamiltonian function:

$$
\begin{aligned}
H= & H_{0}+H_{1}+H_{2}, \\
H_{0}= & a_{1} J_{1}+a_{2} J_{2}+a_{3} J_{3}, \\
H_{1}= & b_{1} J_{1}^{1 / 2} J_{3} \cos \left(\phi_{1}-2 \phi_{3}\right), \\
H_{2}= & b_{2} J_{1} J_{2} \cos 2\left(\phi_{1}-\phi_{2}\right)+b_{3} J_{1}^{2}+b_{4} J_{1} J_{2} \\
& +b_{5} J_{2}^{2}+b_{6} J_{1} J_{3}+b_{7} J_{2} J_{3}+b_{8} J_{3}^{2},
\end{aligned}
$$

where the coefficients are given by

$$
\begin{aligned}
& a_{1}=\Omega_{s}+\frac{\beta_{12}}{2} \quad b_{1}=\sqrt{2} \beta_{s b} \\
& a_{2}=\Omega_{s}-\frac{\beta_{12}}{2} \quad b_{2}=\alpha_{s}-\frac{\varepsilon_{s s}}{2}+\frac{\beta_{22}}{2} \\
& a_{3}=\Omega_{b} \quad b_{3}=\frac{\alpha_{s}}{2}+\frac{\varepsilon_{s s}}{4}+\frac{\lambda^{\prime}}{2}+\frac{\beta_{22}}{4} \\
& b_{4}=2 \alpha_{s}-\beta_{22} \\
& b_{5}=\frac{\alpha_{s}}{2}+\frac{\varepsilon_{s s}}{4}-\frac{\lambda^{\prime}}{2}+\frac{\beta_{22}}{4} \\
& b_{6}=\varepsilon_{s b}+\frac{\lambda^{\prime \prime}}{2} \\
& b_{7}=\varepsilon_{s b}-\frac{\lambda^{\prime \prime}}{2} \\
& b_{8}=\alpha_{b} .
\end{aligned}
$$

The Polyad number is expressed analogously:

$$
P=2\left(J_{1}+J_{2}\right)+J_{3} .
$$

The system in this form also exhibits $1: 1$ and $2: 1$ resonance couplings, but it is no more symmetric with respect to the indices. However we have reduced the number of terms in the Hamiltonian and we are left with a diagonal quadratic part. Actually, this form corresponds to the so-called "normal mode" Hamiltonian by Baggott [1988].

We can obtain integrable limits of this Hamiltonian if we neglect either the coefficient $b_{1}$ or $b_{2}$, i.e. when only one resonance coupling is considered. In this case, the system can be reduce to a 1-dof Hamiltonian, therefore integrable. For instance, the truncated Hamiltonian $\mathrm{H}=\mathrm{H}_{0}+\mathrm{H}_{2}$ is completely integrable with first integrals given by $H, J_{3}$ and $J_{1}+J_{2}$, this is what we expect for the case of a single resonance.

To reduce the system to 2 degrees of freedom, we use the symplectic linear transformation $(J, \phi) \rightarrow(N, \psi)$ defined by

$$
\psi=U \phi, \quad N=\left(U^{T}\right)^{-1} J,
$$


where

$U=\left(\begin{array}{rrr}1 & -1 & 0 \\ 1 & 0 & -2 \\ 0 & 0 & 1\end{array}\right), \quad\left(U^{T}\right)^{-1}=\left(\begin{array}{rrr}0 & -1 & 0 \\ 1 & 1 & 0 \\ 2 & 2 & 1\end{array}\right)$

We obtain $N_{3}=P$, the Polyad number. Then we are left with a 2-degree-of-freedom system in the variables $\left(N_{1}, N_{2}, \psi_{1}, \psi_{2}\right)$. The Hamiltonian can be written as

$$
\begin{aligned}
H= & H_{0}+H_{1}+H_{2} \\
H_{0}= & \alpha_{1} N_{1}+\alpha_{2} N_{2}+\alpha_{3}, \\
H_{1}= & \beta_{1} \sqrt{N_{1}+N_{2}}\left(-2 N_{2}+P\right) \cos \psi_{2}, \\
H_{2}= & -\beta_{2}\left(N_{1}^{2}+N_{1} N_{2}\right) \cos 2 \psi_{1}+\beta_{3} N_{1}^{2} \\
& +\beta_{4} N_{1} N_{2}+\beta_{5} N_{2}^{2} .
\end{aligned}
$$

Note that since $J_{1}>0$ and $J_{2}, J_{3} \geq 0$, it turns out that the feasible region in the new variables is given by $0 \leq-N_{1}<N_{2} \leq P / 2$.

\section{Appendix B}

\section{Frequency map of the Baggott Hamiltonian}

The evolution in time of the fundamental frequencies associated to classical trajectories was obtained with the following numerical procedure.

1. Grid of initial conditions.

We choose a particular slice in the phase space:

$$
\begin{aligned}
& P=34.5, \\
& \theta_{1}=\theta_{1}^{0}, \quad \theta_{2}=\theta_{2}^{0}, \quad \theta_{3}=\theta_{3}^{0}, \\
& 0<I_{1} \leq P / 2, \quad 0<I_{2} \leq P / 2, \\
& I_{1}+I_{2} \leq P / 2, \\
& I_{3}=P-2\left(I_{1}+I_{2}\right) .
\end{aligned}
$$

The initial conditions are evenly spaced in the coordinates $\left(I_{1}, I_{2}\right)$ in the form:

$$
\begin{aligned}
& I_{1}^{0}=0.1 * k, \quad k=1, \ldots, 172 \\
& I_{2}^{0}=0.1 * m+.01, \quad m=1, \ldots, 172-k .
\end{aligned}
$$

2. Numerical integration of the trajectories.

For each initial condition, the trajectory is numerically integrated over a time interval $[0, T]$,
$T=5.7$. We use a Runge-Kutta method of order 7-8, with automatic step-size control (approx. 100000 iterations.) The trajectory is expressed in complex coordinates:

$$
z_{j}=\sqrt{2 I_{j}} e^{i \theta_{j}}, \quad j=1,2,3 .
$$

The step-size is small to ensure small error of the integration method. For the computation of the frequency, the complex time series $z_{1}, z_{2}, z_{3}$ are sampled leaving their lengths of approximately 10000 points not equally spaced in time. This sampling does not have an effect on the accuracy of the method, and reduces the computational time due to less multiplications in the evaluation of the wavelet transform.

3. Computation of the frequency.

For each time series $z_{1}, z_{2}$ and $z_{3}$, we compute a series corresponding to the evolution in time of the fundamental frequencies $\omega_{1}, \omega_{2}$ and $\omega_{3}$. The frequency is obtained from the scale producing the maximum modulus of the wavelet transform at each time point. This was done with the routine Wavelet.

\section{Description of the Wavelet routines}

\section{Routine Wavelet.}

For each time point $\mathrm{b}$ :

First it computes two values of the scale a1, a2 that approximate the largest modulus of the wavelet transform in a range of scales. It calls the function abswav to evaluate the modulus of the wavelet transform at given values of time $b$ and scale $a$.

a1 and a2 are the input for the subroutine fmnbrak that brackets the maximum of abswav.

Then the subroutine fmaxim isolates the scale a that produces the maximum of abswav.

This scale is related to the frequency of $\mathbf{z}$ about the time $\mathrm{b}$ by $\omega=$ cons $/ a$.

The frequency is stored in omega. The information of omega( $j)$ is used to obtain omega $(j+1)$, accelerating the procedure.

\section{input:}

ti, array that holds the time points of the time series, 
z, array containing a complex time series evaluated at each time

point in $t i: z(j)$ is a point of the time series that corresponds to

time $t i(j)$,

$\mathrm{n}$, number of points in the time series.

\section{output:}

omega, array containing the frequency value at each point $b$ in $t i$.

\section{parameters:}

cons, constant that relates the frequency with the scale: $\omega=$ cons $/ a$.

Function Abswav. It computes the modulus of the continuous wavelet transform of $z$ :

$$
\begin{aligned}
L_{\psi} z(a, b) & =\left\langle z, \psi_{a, b}\right\rangle \\
& =a^{-1 / 2} \int_{-\infty}^{\infty} z(t) \bar{\psi}\left(\frac{t-b}{a}\right) d t .
\end{aligned}
$$

The Morlet-Grossman wavelet is

$$
\psi(t)=\frac{1}{\sigma \sqrt{2 \pi}} e^{2 \pi i \lambda t} e^{-t^{2} / 2 \sigma^{2}} .
$$

The modulus of the continuous wavelet transform is computed at a given scale a and time $\mathrm{b}$, for the complex time series $\mathbf{z}$ evaluated at the points in ti. The subroutine uses the Simpson formula for numerical integration of the product between $z$ and $\bar{\psi}_{a b}$, over the time interval $[b-3 \sqrt{2} \sigma a, b+3 \sqrt{2} \sigma a]$ (effective support of the Morlet-Grossman wavelet).

\section{input:}

a, scale,

$\mathrm{b}$, time,

ti, $z, n$, time points, values and number of points of the time series.

output:

abswav, modulus of the wavelet transform.

\section{parameters:}

sigma, lambda, variance and frequency of the Morlet-Grossman wavelet.

\section{Subroutine fmnbrak.}

Routine that brackets the maximum of a function. Given two initial values $a_{1}, a_{2}$, the routine searches for the uphill direction and returns new points $a_{1}, a_{2}, a_{3}$ that bracket the maximum (i.e. $a_{1}<a_{2}<a_{3}$ and $f\left(a_{2}\right)$ is greater than both $f\left(a_{1}\right)$ and $\left.f\left(a_{3}\right)\right)$.

It is used to find points of scale a that bracket the maximum of abswav at each time $b$.

\section{input:}

a1, a2, initial values of the scale $a$, b, time, ti, $z, n$, time points, values and number of points of the time series.

\section{output:}

a1, a2, a3, points of the scale that bracket the maximum, $f 1, f 2, f 3$, evaluation of abswav at the points a1, a2, a3.

\section{Subroutine fmaxim.}

Routine that finds the maximum of a function given three abscissas that bracket the maximum. This routine isolates the maximum to a fractional precision using Brent's method.

It finds the scale a producing the maximum modulus of the wavelet transform abswav for the time series $\mathbf{z}$ at each time point $\mathrm{b}$ with tolerance $10^{-8}$.

input:

a1, a2, a3, points that bracket the maximum,

$\mathrm{b}$, time, ti, $z, n$, time points, values and number of points of the time series.

output:

$\mathrm{x}$, point where the maximum is located, $\mathrm{fx}$, value of the maximum. 